\title{
Coherence in Dense Cores. II. The Transition to Coherence
}

\section{Citation}

Goodman, Alyssa A., Joseph A. Barranco, David J. Wilner, and Mark H. Heyer. 1998. "Coherence in Dense Cores. II. The Transition to Coherence." The Astrophysical Journal 504 (1): 223-46. https://doi.org/10.1086/306045.

\section{Permanent link}

http://nrs.harvard.edu/urn-3:HUL.InstRepos:41397452

\section{Terms of Use}

This article was downloaded from Harvard University's DASH repository, and is made available under the terms and conditions applicable to Other Posted Material, as set forth at http:// nrs.harvard.edu/urn-3:HUL.InstRepos:dash.current.terms-of-use\#LAA

\section{Share Your Story}

The Harvard community has made this article openly available.

Please share how this access benefits you. Submit a story.

Accessibility 
The Astrophysical Journal, 504:223-246, 1998 September 1

(C) 1998. The American Astronomical Society. All rights reserved. Printed in U.S.A.

\title{
COHERENCE IN DENSE CORES. II. THE TRANSITION TO COHERENCE
}

\author{
Alyssa A. Goodman ${ }^{1}$ \\ Harvard University Department of Astronomy, Cambridge, MA 02138; agoodman@cfa.harvard.edu \\ JOSEPH A. BARRANCO \\ Astronomy Department, University of California, Berkeley, Berkeley, CA 94720; barranco@ucbast.berkeley.edu \\ DAVID J. WILNER \\ Harvard-Smithsonian Center for Astrophysics, 60 Garden Street, Cambridge, MA 02138; dwilner@cfa.harvard.edu \\ AND \\ MARK H. HeYER \\ Five College Radio Astronomy Observatory, University of Massachusetts, Amherst, MA 01003; heyer@fcrao1.phast.umass.edu \\ Received 1997 June 17; accepted 1998 February 5
}

\begin{abstract}
After studying how line width depends on spatial scale in low-mass star-forming regions, we propose that "dense cores" (Myers \& Benson 1983) represent an inner scale of a self-similar process that characterizes larger scale molecular clouds.

In the process of coming to this conclusion, we define four distinct types of line width-size relation $\left(\Delta v \propto R^{a_{i}}\right)$, which have power-law slopes $a_{1}, a_{2}, a_{3}$, and $a_{4}$, as follows: Type 1 -multitracer, multicloud intercomparison; Type 2-single-tracer, multicloud intercomparison; Type 3-multitracer study of a single cloud; and Type 4 - single-tracer study of a single cloud. Type 1 studies (of which Larson 1981 is the seminal example) are compendia of Type 3 studies which illustrate the range of variation in the line width-size relation from one region to another.

Using new measurements of the $\mathrm{OH}$ and $\mathrm{C}^{18} \mathrm{O}$ emission emanating from the environs of several of the dense cores studied in $\mathrm{NH}_{3}$ by Barranco \& Goodman (1998; Paper I), we show that line width increases with size outside the cores with $a_{4} \sim 0.2$. On scales larger than those traced by $\mathrm{C}^{18} \mathrm{O}$ or $\mathrm{OH},{ }^{12} \mathrm{CO}$ and ${ }^{13} \mathrm{CO}$ observations indicate that $a_{4}$ increases to $\sim 0.5$ (Heyer \& Schloerb 1997). By contrast, within the half-power contour of the $\mathrm{NH}_{3}$ emission from the cores, line width is virtually constant, with $a_{4} \sim 0$. We interpret the correlation between increasing density and decreasing Type 4 power-law slope as a "transition to coherence." Our data indicate that the radius $R_{\text {coh }}$ at which the gas becomes coherent (i.e., $a_{4} \rightarrow 0$ ) is of order $0.1 \mathrm{pc}$ in regions forming primarily low-mass stars. The value of the nonthermal line width at which "coherence" is established is always less than but still of order of the thermal line width of $\mathrm{H}_{2}$. Thus coherent cores are similar to, but not exactly the same as, isothermal balls of gas.

Two other results bolster our proposal that a transition to coherence takes place at $\sim 0.1 \mathrm{pc}$. First, the $\mathrm{OH}, \mathrm{C}^{18} \mathrm{O}$, and $\mathrm{NH}_{3}$ maps show that the dependence of column density on size is much steeper $\left(N \propto R^{-0.9}\right)$ inside $R_{\text {coh }}$ than outside of it $\left(N \propto R^{-0.2}\right)$, which implies that the volume filling factor of coherent cores is much larger than in their surroundings. Second, Larson (1995) has recently found a break in the power law characterizing the clustering of stars in Taurus at 0.04 pc, just inside of $R_{\text {con }}$. Larson and we interpret this break in slope as the point at which stellar clustering properties change from being determined by the (fractal) gas distribution (on scales greater than $0.04 \mathrm{pc}$ ) to being determined by fragmentation processes within coherent cores (on scales less than $0.04 \mathrm{pc}$ ).

We speculate that the transition to coherence takes place when a dissipation threshold for the MHD turbulence that characterizes the larger scale medium is crossed at the critical inner scale $R_{\text {coh }}$. We suggest that the most likely explanation for this threshold is the marked decline in the coupling of the magnetic field to gas motions due to a decreased ion/neutral ratio in dense, high filling factor gas.

Subject headings: ISM: clouds - ISM: kinematics and dynamics - ISM: structure - line: profiles
\end{abstract}

\section{INTRODUCTION}

Low-mass $\left(\sim 1-10 M_{\odot}\right)$ dense cores appear to be relative islands of calm in a more turbulent sea. These cores, which are the birthplace of many of the stars in our Galaxy, have average density $\sim 10^{4} \mathrm{~cm}^{-3}$ and size of approximately a few tenths of a parsec, and are characterized by very small internal velocity dispersions of approximately several tenths of a kilometer per second (see Myers \& Benson 1983; Benson \& Myers 1989). Barranco \& Goodman (1998; hereafter, Paper I) present high-sensitivity $\mathrm{NH}_{3}$ mapping of dense cores, and this paper puts those observations into context by compar-

\footnotetext{
${ }^{1}$ National Science Foundation Young Investigator.
}

ing the $\mathrm{NH}_{3}$ observations with $\mathrm{C}^{18} \mathrm{O}$ and $\mathrm{OH}$ observations of the core environments. Taken together, the two studies show that a line width-size power-law scaling relation similar to the one discovered more than a decade ago by Larson (1981) appears to have a break in slope at a size scale roughly comparable to the FWHM contour of an $\mathrm{NH}_{3} \operatorname{map}(\sim 0.1 \mathrm{pc})$.

Larson (1981) discovered empirical correlations among line width, cloud mass, volume density, and cloud "size" by extracting a single value for each quantity from a single spectral line map of a single "cloud" or clump and then plotting line width as a function of size, line width as a function of mass, and density as a function of size. Larson's compilation included data from spectral line maps of about 
TABLE 1

Velocity Dispersion Terminology Summary

\begin{tabular}{|c|c|c|}
\hline Symbol & Context & Description \\
\hline$\sigma_{\text {obs }} \ldots \ldots$ & Observed quantity & Observed velocity dispersion \\
\hline$\sigma_{T} \ldots \cdots$ & $\sigma_{T}=\sqrt{k T / m_{\mathrm{tr}}}$ & $\begin{array}{l}\text { Thermal velocity dispersion (sound speed in } \\
\text { isothermal gas at temperature } T \text {, of particles } \\
\text { with mass } m_{\mathrm{tr}} \text { ) }\end{array}$ \\
\hline$\sigma_{\mathrm{NT}} \cdots \cdots$ & $\sigma_{\mathrm{obs}}^{2}=\sigma_{T}^{2}+\sigma_{\mathrm{NT}}^{2}$ & Nonthermal velocity dispersion \\
\hline$\sigma_{\text {int }} \cdots \cdots$ & See Paper I & $\begin{array}{l}\text { Intrinsic velocity dispersion, after effects of } \\
\text { hyperfine blending have been removed (see } \\
\text { Paper I) }\end{array}$ \\
\hline$\sigma_{0} \ldots \ldots$ & $\sigma_{\mathrm{NT}}^{2}=\sigma_{0}^{2}\left[1+\left(R / R_{0}\right)^{2 a}\right]$ & $\begin{array}{l}\text { Asymptotic value of nonthermal velocity } \\
\text { dispersion approached by } \mathrm{NH}_{3} \text { observations }\end{array}$ \\
\hline
\end{tabular}

50 different regions. The maps were made using many different molecular lines which trace gas in the density range from $\sim 10$ to $10^{5} \mathrm{~cm}^{-3}$ over size scales from $\sim 0.1$ to $100 \mathrm{pc}$, with resolution typically at least 10 times smaller than the map extent. In all but the smallest clouds in Larson's study, the observed (one-dimensional) velocity dispersion $\sigma_{\mathrm{obs}}$ far exceeds the (one-dimensional) thermal velocity dispersion $\sigma_{T}=\left(k T / m_{\mathrm{tr}}\right)^{1 / 2}$, where $T$ is kinetic temperature and $m_{\mathrm{tr}}$ is the mass of the tracer species observed. Larson fitted power laws by eye to the line width-size and line width-mass correlations and ultimately found

$$
\begin{gathered}
\Delta v_{\text {obs }} \propto R^{a} \quad a \approx 0.38, \\
n \propto R^{b} \quad b \approx-1.1,
\end{gathered}
$$

where $\Delta v_{\text {obs }}=2.35 \sigma_{\text {obs }}$ is the observed FWHM line width, ${ }^{2}$ $n$ is particle density, and $R$ is the diameter of a particular (e.g., $50 \%$ ) contour in a spectral line map. Larson also showed that the clouds in his study were close to virial equilibrium, in that

$$
\Delta v_{\mathrm{obs}}=\alpha\left(\frac{G M}{R}\right)^{0.5},
$$

where $M$ is the total mass with density $n$ within $R$, and $\alpha$ is a constant of order unity which depends on cloud shape. If $a=0.5$ and $b=-1$ then any one of equations (1), (2), or (3) can be derived from the other two.

Larson (1981) also examined the variations in line width within individual clouds. To do this, he compared concentric spectral line maps of various density tracers for a number of regions. In so doing, he found a correlation similar to equation (1). More recent studies that use the multitracer approach to investigate line width-size scaling in detail within individual clouds give a range of values for $0.2 \lesssim a \lesssim 0.7$ and indicate that massive star-forming regions tend toward the lower values of $a$ (e.g., Caselli \& Myers 1995).

Larson pointed out that $a=0.38$ is very close to the value $a=0.33$ that is predicted for a turbulent flow in incompressible gas (Kolmogorov 1941), but he noted that good agreement of these two exponents is not necessarily expected, since molecular clouds are compressible and self-

\footnotetext{
${ }^{2}$ Table 1 presents a summary of the velocity dispersion notation used in this paper. In all cases, the full width at half maximum (FWHM), $\Delta v$, of a Gaussian line is equal to $(8 \ln 2)^{1 / 2} \sigma=2.35 \sigma$, where $\sigma$ is the dispersion characterizing a Gaussian proportional to $\exp \left(-v^{2} / 2 \sigma^{2}\right)$.
}

gravitating. In fact, the ISM is also magnetized, and a theoretical value of $a$ for a magnetized, compressible gas is not readily derived, since it depends on field geometry and the gas equation of state.

The correlations that Larson discovered have come to be known as Larson's Laws, and they have become the subject of intense study (e.g., Myers 1983; Fleck 1988; Myers \& Goodman 1988b, hereafter MG88b; Henriksen 1991; Falgarone, Puget, \& Pérault 1992; Fuller \& Myers 1992; Miesch \& Bally 1994; Caselli \& Myers 1995). Physically, no complete explanation of Larson's Laws has yet been established. Turbulence (e.g., Falgarone \& Phillips 1990; Henriksen 1991; Falgarone et al. 1994; Lis et al. 1996) and/or an array of magnetohydrodynamic waves (e.g., Arons \& Max 1975; Zweibel \& Josafatsson 1983; Carlberg \& Pudritz 1991; McKee \& Zweibel 1995; Gammie \& Ostriker 1996) remain the leading hypotheses, but the ultimate origin and exact nature of the "suprathermal" kinetic energy is still unclear.

Magnetohydrodynamic scenarios gain observational support from field strength measurements in regions with $5<n<10^{10} \mathrm{~cm}^{-3}$ which usually indicate rough equipartition between magnetic and kinetic energy (Myers \& Goodman 1988a; Goodman et al. 1989; Crutcher et al. 1993; Heiles et al. 1993, and references therein; Goodman \& Heiles 1994; Myers et al. 1995). Recent two-dimensional numerical simulations of MHD turbulence also indicate this kind of equipartition (Passot et al. 1995). Nonmagnetic turbulence models can produce line profiles not unlike those observed (Falgarone et al. 1994; Dubinski, Narayan, $\&$ Phillips 1995) and scaling laws similar to equation (1), but the structural properties and lifetimes of these models have yet to be compared systematically with observations. It is likely that magnetic fields are necessary to maintain the effects of turbulence-generating processes over longer timescales (see Gammie \& Ostriker 1996 and references therein).

For the purposes of this paper, we put forward the hypothesis that Larson's Laws as stated in equation (1) and (2) are indicative of some kind of turbulent regime in the ISM. We expect that magnetic fields play a key role in mitigating this turbulence and that the velocity field is actually a superposition of magnetic waves undergoing strongly nonlinear interactions which can also be thought of as MHD turbulence.

What is of special interest to us in this paper is what happens to Larson's Laws on small size scales. Since the observed line width $\Delta v_{\text {obs }}$ cannot go below the thermal line width of the tracer observed, $\Delta v_{T}=2.35 \sigma_{T}$, it is obvious that the correlation between line width and cloud size must end at some small size (i.e., line width cannot decrease intermi- 
nably with cloud size). Myers (1983) reformulated Larson's Laws to take this effect into account. He denoted the line width as the quadrature sum of a nonthermal and a thermal component,

$$
\Delta v_{\mathrm{obs}}^{2}=\Delta v_{\mathrm{NT}}^{2}+\Delta v_{T}^{2},
$$

so that the nonthermal component of the line width, $\Delta v_{\mathrm{NT}}$, does not depend on the mass of the tracer used in the observation. In this formulation, the nonthermal motions can be equated with macroscopic motions, while the thermal line width accounts for microscopic motions. For virtually all of the clouds in Larson's original study, the thermal line width comprises an insignificant fraction of the total line width. Low-mass dense cores, ${ }^{3}$ however, represent a regime in which thermal and nonthermal line width are roughly equal.

In this paper, we investigate the transition from the "nonthermal" to the "thermal" regime in detail, using new high-sensitivity maps of low-mass dense cores (Paper I) and their environs (§ 2). Specifically, we find an apparent decrease in the slope of line width-size relations derived from single-tracer maps of individual regions that we can associate with this transition (§ 4). In the nonthermal regime, this slope is always positive (so that line width increases with size), but in the thermal region, the observed line width is approximately constant (i.e., independent of scale). However, this nearly constant width in the thermal regime is always measurably higher than the true thermal line width, $\sigma_{t}=\left(k T_{K} / m_{\text {tracer }}\right)^{1 / 2}$, where $T_{\mathrm{K}}$ is the measured kinetic temperature and $m_{\text {tracer }}$ is the mass of the species observed. In fact, no observations in this paper, or others of which we know, show a purely thermal line width in a dense core. We suggest that the regions of nearly constant line width, while not purely thermal, are still physically distinguished from their surroundings in that they are "coherent," and we suggest ways in which these coherent cores might arise $(\S 5)$.

\section{OBSERVATIONS}

\section{1. $\mathrm{C}^{18} \mathrm{O}$ Observations}

We observed the $J=1-0$ transition of $\mathrm{C}^{18} \mathrm{O}$ using the QUARRY 15 beam array at the Five College Radio Astronomy Observatory ${ }^{4}$ (FCRAO) $14 \mathrm{~m}$ radio telescope in 1994 June and November and 1995 February. The 1024 channel autocorrelation spectrometer gave a resolution of $0.016 \mathrm{~km} \mathrm{~s}^{-1}$ sampled at $0.013 \mathrm{~km} \mathrm{~s}^{-1}$, Hanning smoothed to a final resolution of $0.018 \mathrm{~km} \mathrm{~s}^{-1}$. The system temperature was typically $600 \mathrm{~K}$, and each spectrum represents about 150 minutes of integration, giving a root mean square (rms) noise of $0.1 \mathrm{~K}$ after Hanning smoothing.

The $\mathrm{C}^{18} \mathrm{O}$ maps presented here are sampled at $50^{\prime \prime}$ intervals, and the FWHM of a single QUARRY/FCRAO beam is $48^{\prime \prime}$, as determined by observations of planets. Single Gaussian fits to each line profile comprising the map were performed in CLASS after removing a first-order baseline. The results of the fitting are discussed further in $\S 3$.

\footnotetext{
${ }^{3}$ All of the data in this paper are for what are often called "low-mass dense cores." Low-mass cores typically form one or a handful of stars each with a mass at most a few solar masses. In higher mass star-forming cores, stars primarily form in large groups and observed line widths are almost always very suprathermal (Lada 1992).

${ }^{4}$ FCRAO is operated by the University of Massachusetts for the National Science Foundation under grant AST 94-20159.
}

\subsection{OH Observations}

The 1665 and $1667 \mathrm{MHz}$ transitions of $\mathrm{OH}$ were mapped at the Arecibo Observatory ${ }^{5}$ in 1989 and 1991. A subset of the data for the B1 cloud has appeared in previous publications, in which the $\mathrm{OH}$ mapping observing setup at Arecibo is described in detail (Goodman et al. 1989; Crutcher et al. 1994). The spatial resolution of the observations is $3^{\prime}$, and the velocity resolution before smoothing was $0.22 \mathrm{~km} \mathrm{~s}^{-1}$. All of the data were Hanning smoothed before Gaussian fits were performed. The typical rms of the B1 spectra, at $0.08 \mathrm{~K}$, is superior to the TMC-1C spectra, which is typically $0.12 \mathrm{~K}$.

\section{3. $\mathrm{NH}_{3}$ Observations}

All of the $\mathrm{NH}_{3}$ data included in this paper are included in Paper I. The observing setup used at the Haystack Observatory ${ }^{6}$ to acquire those data is described in detail there. Here, we will just reiterate that the data near the edges of the maps represent very long integration observations (typically several hours per point) with rms noise $\sim 0.04 \mathrm{~K}$, giving signal-to-noise ratios of at least 3 at any one point. The velocity resolution of the $\mathrm{NH}_{3}$ spectra ranges from 0.04 to $0.08 \mathrm{~km} \mathrm{~s}^{-1}$, and the spatial resolution ranges from $60^{\prime \prime}$ to $80^{\prime \prime}$ (see Paper I).

\section{SPECTRAL LINE DATA AND MAPS}

It is difficult to make use of all of the information in a spectral line map simultaneously (see, for example, Scalo 1984; Pérault, Falgarone, \& Puget 1986; Kleiner \& Dickman 1987; Stutzki \& Güsten 1990; Miesch \& Bally 1994; Williams, de Geus, \& Blitz 1994; Heyer \& Schloerb 1997). Often, only peak antenna temperature or integrated intensity are contoured in print, and much information is hidden. The analysis in this section is an attempt to display and understand some of the "hidden" velocity information in maps of the $n \gtrsim 10^{3} \mathrm{~cm}^{-3}$ gas around dense cores. We utilize both the $J=1-0$ line of $\mathrm{C}^{18} \mathrm{O}$ and the $1667 \mathrm{MHz}$ line of $\mathrm{OH}$ as tracers of roughly this density.

Figures $1 a, 1 b$, and 2 all show gray-scale and contour maps of antenna temperature in the left-hand panel and a gray-scale map of line width with representative antenna temperature contours superposed in the right-hand panel. The three figures, which show data for B1, TMC-1C, and L1251A, respectively, exhibit one striking similarity: line width appears to decrease toward the peaks of the map and increase in the valleys. In all three cases, the line profiles are well approximated by Gaussians throughout the map, and the line parameters plotted in all figures in this paper are based on Gaussian fits to spectra. In $\S 4$, we show that the qualitative tendency for line width to decrease with increasing antenna temperature apparent in the figures can be transformed into a quantitative line width-size relation.

It is not always the case that a single Gaussian component can be used to represent the gas distribution associated with the $n \sim 10^{3} \mathrm{~cm}^{-3}$ environment of dense cores. In

\footnotetext{
${ }^{5}$ The Arecibo telescope is operated by Cornell University for the National Science Foundation, under the auspices of the NAIC.

${ }^{6}$ Haystack Observatory is operated by the Massachusetts Institute Technology, on behalf of NEROC, and is funded by the National Science Foundation.
} 


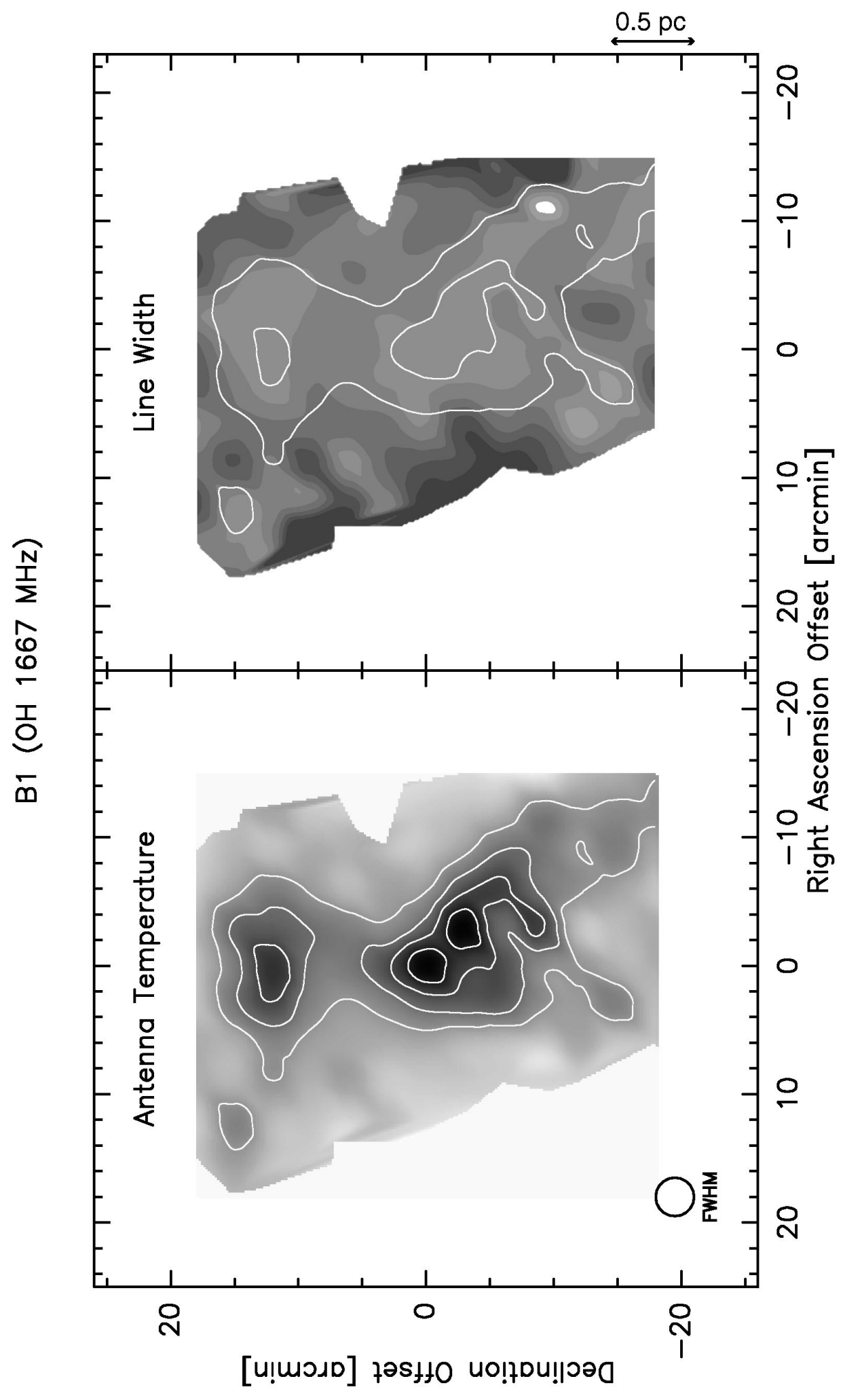

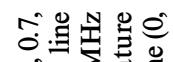

ก๊

원영

题起要

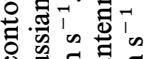

o. 명

Q

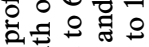

要这

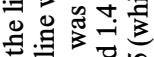

류유.

过记

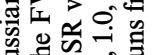

F

๘

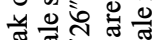

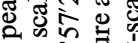

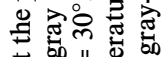

舟

承

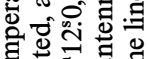

㐘

包类

击

웅

면

ฮิ



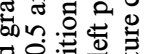

范实造

范家白

记

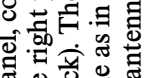

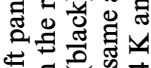

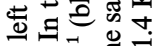

g $x^{\prime}=\overrightarrow{0}$

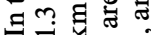

唡

घ0ㅇㅇํㅇ

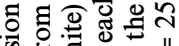

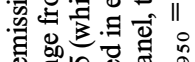

춘웡

远过

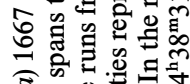

उิ

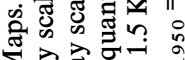

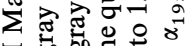

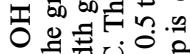

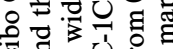

可高造

过星品

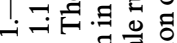

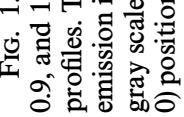




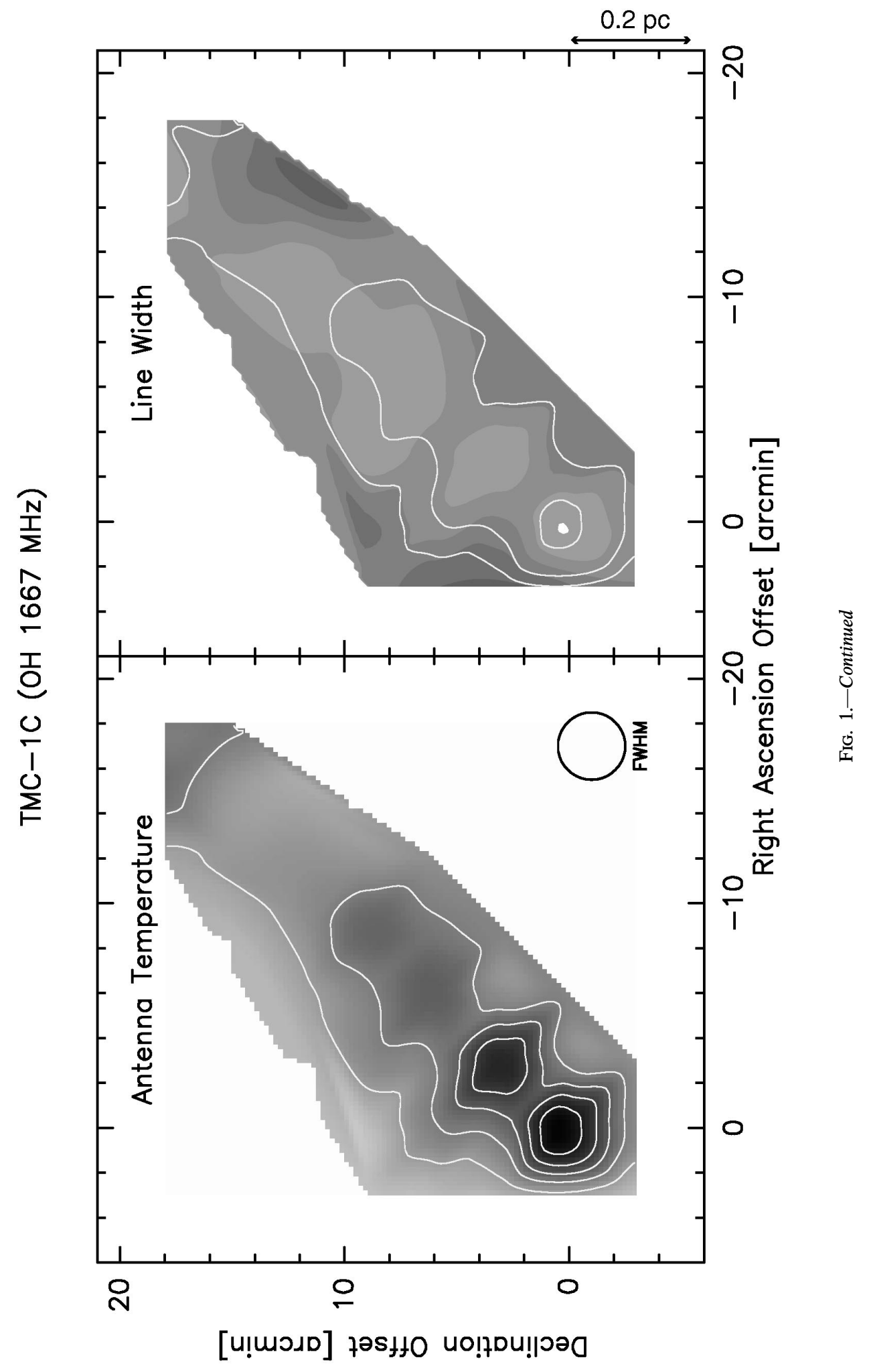




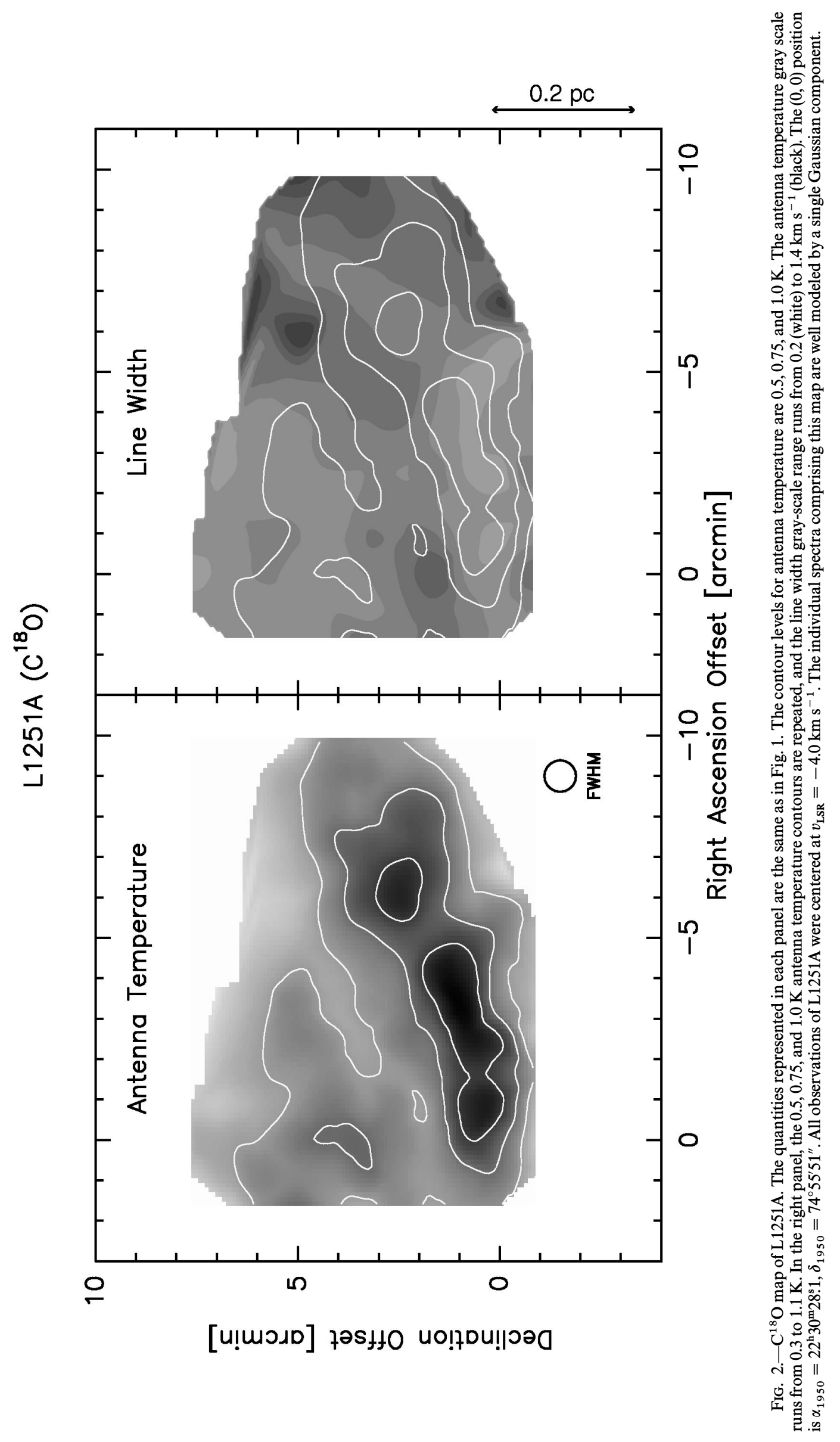




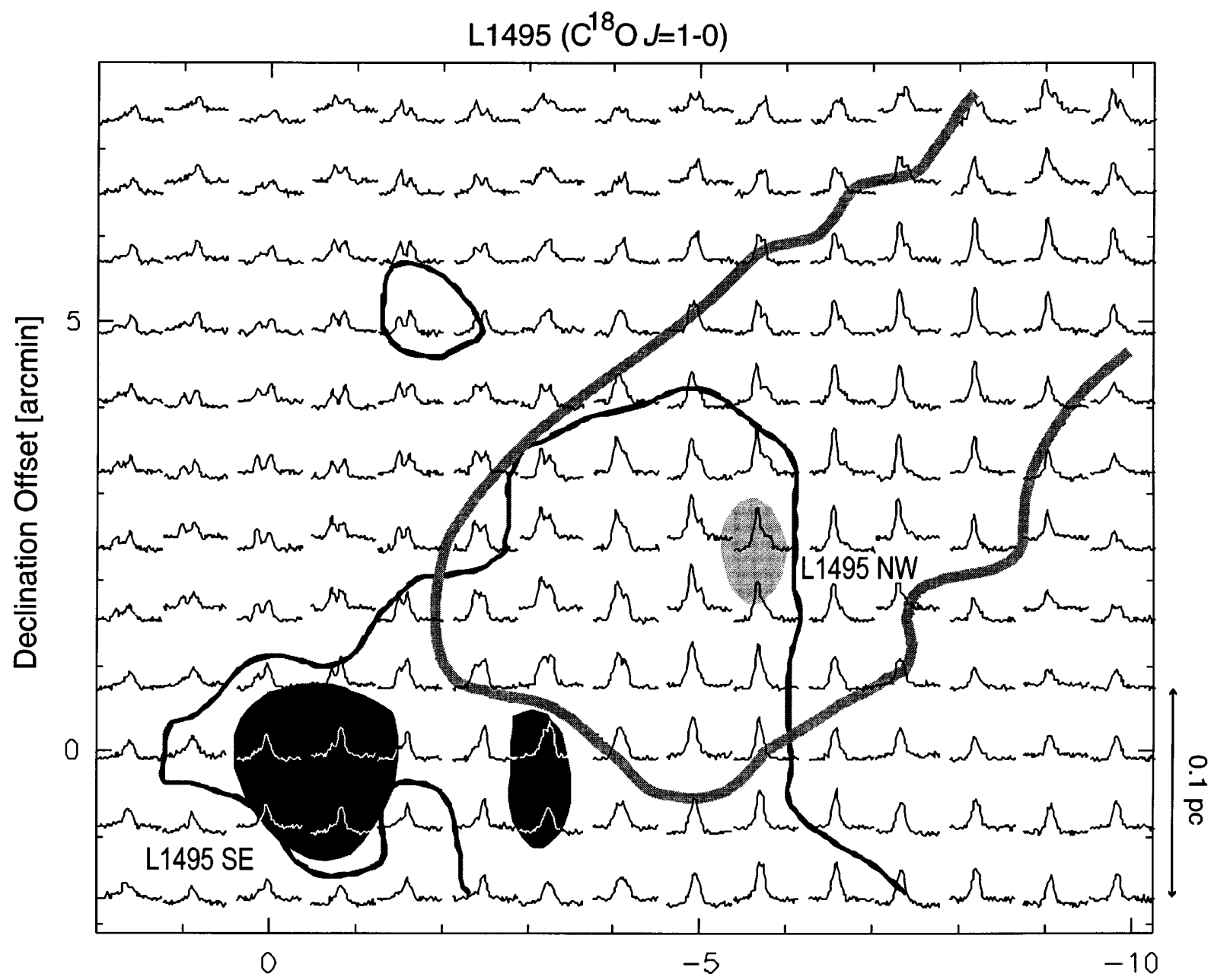

Right Ascension Offset [arcmin]

FIG. 3. $-\mathrm{C}^{18} \mathrm{O}$ map of $\mathrm{L} 1495$. At each position, a $\mathrm{C}^{18} \mathrm{O}$ spectrum is shown. The $(0,0)$ position is $\alpha_{1950}=4^{\mathrm{h}} 11^{\mathrm{m}} 10^{\mathrm{s}} .0, \delta_{1950}=28^{\circ} 01^{\prime} 58^{\prime \prime}$, and all observations of L1495 were centered at $v_{\mathrm{LSR}}=6.4 \mathrm{~km} \mathrm{~s}^{-1}$. The lighter single contour superposed on the spectra shows the $0.52 \mathrm{~K} \mathrm{~km} \mathrm{~s}{ }^{-1}$ contour of integrated $\mathrm{C}^{18} \mathrm{O}$ emission from 5.2 to $6.6 \mathrm{~km} \mathrm{~s}^{-1}$, while the darker contours show the same contour level for integrated emission from 6.6 to $8 \mathrm{~km} \mathrm{~s}^{-1}$. These two distinct velocity ranges are associated with the LSR velocities of the $\mathrm{NH}_{3}$ cores L1495SE and L1495NW, respectively. A shaded $0.4 \mathrm{~K}$ contour (from Goodman et al. 1993) for each of the $\mathrm{NH}_{3}$ cores is shown and labeled (note that both southeast "pieces" belong to L1495SE). A casual glance at the figure shows pervasive double-peaked and asymmetric $\mathrm{C}^{18} \mathrm{O}$ line profiles. Prolonged staring at the figure further shows the intensity-weighted peak velocity of the emission shifting blueward, along the direction from L1495SE to L1495NW.

Figure 3, we show the result of extensive $\mathrm{C}^{18} \mathrm{O}$ observations of the gas around the dense cores in L1495. $\mathrm{NH}_{3}$ observations of L1495 reveal two cores, L1495SE and L1495SW, at velocities separated by more than an $\mathrm{NH}_{3}$ line width, within the boundaries of the region mapped in $\mathrm{C}^{18} \mathrm{O}$. In $\mathrm{C}^{18} \mathrm{O}$, the same two distinct velocity components are apparent, but the extent to which they blend together in velocity space (owing to the larger line width in $\mathrm{C}^{18} \mathrm{O}$ relative to $\mathrm{NH}_{3}$ ) makes it dangerous to try to assign some $\mathrm{C}^{18} \mathrm{O}$ emission to one core and the rest to another. This situation is not uncommon in spectral line maps of regions crowded with star-forming cores. The dense cores traced by $\mathrm{NH}_{3}$ are usually easily identifiable entities (with single-component, Gaussian lines), but the lower the density one probes, the more velocity blending becomes a problem, even when very high spectral resolution is used. For the analysis that follows, we restrict our study to less crowded regions, such as B1, TMC-1C, and L1251A. For each of these regions, an $\mathrm{NH}_{3}$ map of the "core" is compared with a map of a lower density tracer $\left(\mathrm{OH}\right.$ or $\left.\mathrm{C}^{18} \mathrm{O}\right)$ of the core's "environment."

\section{CORRELATIONS AND LINE WIDTH-SIZE RELATIONS}

\subsection{Line Width-Size Relations in Context}

There are several different ways spectral line maps can be used to measure how the velocity dispersion of gas changes as a function of the size scale sampled, including constructing so-called line width-size relations. Below, we list four distinct "Types" of line width-size relations that can be studied observationally, and in $\S 5$ we discuss the subtle differences among the Types. The power-law slope of log $\Delta v_{\mathrm{NT}}-\log R$ is given as a symbol at the end of each description. Table 2 and Figure 9 provide a summary of the

TABLE 2

TyPes of Line WiDTh-Size ReLATION

\begin{tabular}{lcc}
\hline \hline & Multiple Tracers & Single Tracer \\
\hline Multiple Clouds & 1 & 2 \\
Single Cloud & 3 & 4 \\
\hline
\end{tabular}


four Types, and periodic reminders of the Types' definitions are included throughout this paper.

Type 1: Multitracer, multicloud.-A power law fitted to line width as a function of size where a single line width is assigned to a single cloud, usually by taking a "representative" 7 line width within a FWHM contour, the extent of which is used as size (e.g., Larson 1981; Myers et al. 1988b; Falgarone et al. 1992; Fuller \& Myers 1992; Caselli \& Myers 1995). Slope $=a_{1}$.

Type 2: Single-tracer, multicloud-Same as Type 1, except that the study is limited to maps in a single spectral line (e.g., Dame et al. 1986; Solomon et al. 1987). Slope = $a_{2}$.

Type 3: Multitracer, single-cloud-Same as Type 1, except that the study is limited to maps of a single region (e.g., Fuller \& Myers 1992; Caselli \& Myers 1995). Slope = $a_{3}$.

Type 4: Single-tracer, single-cloud-Different from Type 1 in that the spatial dependence of line width on size within a single map of a single region is studied (e.g., Miesch \& Bally 1994; Heyer \& Schloerb 1997; Paper I). The remainder of this section is devoted to deriving this relation for $\mathrm{OH}, \mathrm{C}^{18} \mathrm{O}$, and $\mathrm{NH}_{3}$ data. Slope $=a_{4}$.

Unfortunately, any of these techniques is limited by the fact that spectral line widths sample only one spatial dimension of a velocity distribution integrated over lines of sight of varying length. One is thus forced to use the distribution of line emission over the two-dimensional plane of the sky (i.e., a spectral line map) as a source of three-dimensional spatial size information (see $\S 4.2 .1$ and Fig. 4). Section 5 includes a discussion of the sensitivity of the four techniques listed above to assumptions about what three-dimensional distribution of gas corresponds to the observed twodimensional projection.

\subsection{Analysis Technique}

To quantitatively relate line width to size scale using the data shown in Figures $1 a, 1 b$, and 2, we perform a two-step analysis. In the first step, we fit for a relationship between antenna temperature and size, and in the second, we fit for a relationship between line width and antenna temperature. Then, by combining these two empirically determined results, we can derive a line width-size relation of Type 4 .

We choose this two-step process over just assigning a size to each measured line width based on its radial distance from the nearest temperature peak because of the clear way in which the two-step process handles maps that contain one principal peak and many subpeaks. For example, consider the map of L1251A shown in Figure 2. Notice the single $0.75 \mathrm{~K}$ contour in the northeast quadrant of the map. The technique we describe in $\S \S 4.2 .1$ and 4.2.2 will assign the line width from this little piece of "chaff" to the $\sim 0.3 \mathrm{pc}$ size scale associated with the dominant $0.75 \mathrm{~K}$ contour that encloses the main peak in the L1251A core. A technique

\footnotetext{
${ }^{7}$ This representative line width is assigned in different ways by different authors. It is often the average line width within the FWHM contour, but it is sometimes the line width at the peak of the map, the average line width at the FWHM contour, or the line width of the sum of all of the spectra within the FWHM contour. As long as the range of line widths and sizes sampled in a Type 1 study is large (e.g., Larson 1981), the exact method used to assign "a" line width and " a" size to each cloud makes little difference in the slope derived-although mixing methods in a single line width-size plot will increase the scatter about the correlation found.
}

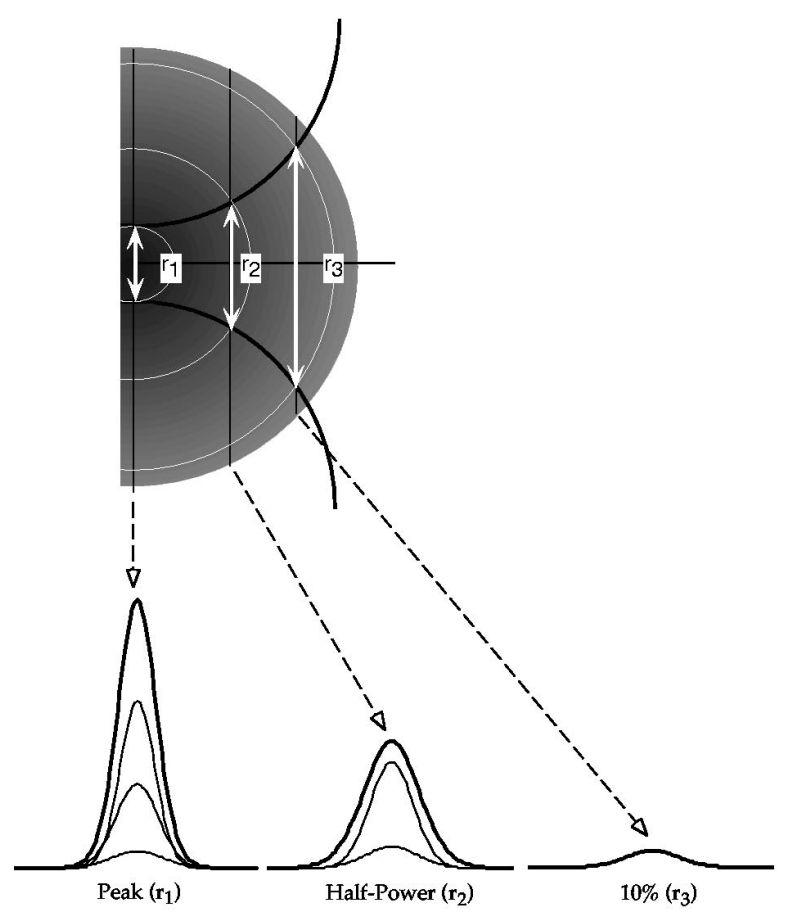

FIG. 4.-Measuring "radius" in a spectral line map. The gray scale at the top depicts a generic centrally condensed density distribution in hypothetical cloud. The three labeled (plane-of-the-sky) length scales, $r_{1}, r_{2}$, and $r_{3}$, mark zones that produce emission near the peak, half-power, and $10 \%$ contours in a map, respectively. The white two-headed arrows show the extent along the line of sight of the gas which produces the bulk of the emission in these zones, and representative spectra for the zones are shown at the bottom. The heavy line in each spectrum shows the total observed spectrum (produced by gas along the entire line of sight through the cloud), and the lighter lines show the contributions from each zone. Notice that the highest density zone sampled along each line of sight has the greatest influence in determining the line width of the summed profile. The precise length of the white arrows depends upon the exact density profile of the cloud and on its velocity structure. Nonetheless, it is apparent from the figure that the progression of antenna temperature downward from the map peak will correspond to an increase in the size scale sampled. As a first approximation, the length of the white arrows can be estimated as similar to the plane-of-the-sky extent of corresponding $T_{A}$ contours (e.g., $r_{1}, r_{2}$, and $r_{3}$ ).

that just measures a radius from the nearest local temperature peak would find the peak inside the small $0.75 \mathrm{~K}$ contour and assign the line width of this little piece of chaff to a very small size scale, $\sim 0.1 \mathrm{pc}$, which is comparable to (but not physically related to) the scale of the darkest black regions around the main peaks in the left-hand panel of Figure 2. Our two-step process is less resolution-dependent than one that relies on measuring the distance from local maxima (i.e., the size of every apparent subclump). It also allows for the resulting "line width-size" relation to be more directly translated to a physically meaningful line width-density relation.

\subsubsection{Antenna Temperature-Size Correlations}

In $\mathrm{C}^{18} \mathrm{O}$ and $\mathrm{OH}$ maps of the emission around dense cores, antenna temperature contours are usually nested and centered roughly on the $\mathrm{NH}_{3}$ peak, so contours of $T_{A}$ can serve as good indicators of size scale. Figure 4 shows a very simple hypothetical condensation and illustrates how antenna temperature is physically related to size scale. The principal uncertainty in "radii" derived from a technique based on the ideas in Figure 4 stems from an inexact know- 
ledge of the condensation's density profile. For the hypothetical profile shown in Figure 4, the lengths of the white arrows (which measure the length scale along the line of sight that is best associated with the measured line width) are quite close to equal to the plane-of-the-sky radii labeled $r_{1}, r_{2}$, and $r_{3}$. For some extreme density profiles, the planeof-the-sky and line-of-sight length scales will not agree as well, but as an approximate method of assigning physically meaningful sizes to map contours, we think this method is a good start - at least for centrally condensed density distributions. One important point to note about this technique of defining size is that it allows for extrapolation into a regime in which the derived " $R$ " is smaller than the beam size. This extrapolation only becomes relevant for points at or very near the map peak, which would be otherwise artificially assigned either $R=0$ or $R=$ beam size in a scheme in which size is just measured radially from the peak.

As shown in Paper I, for $\mathrm{NH}_{3}$ core maps, fitting the antenna temperature projected size data typically gives $T_{A} \propto R^{-1}$. For the $\mathrm{C}^{18} \mathrm{O}$ maps and $\mathrm{OH}$ maps, the dependence of antenna temperature on size has a markedly shallower slope. Figure 5 shows power-law fits to $T_{A}-R$ relations for the $\mathrm{OH}$ maps of $\mathrm{B} 1$ and TMC-1C and for the $\mathrm{C}^{18} \mathrm{O}$ map of L1251A. The points fit were derived by assigning a size equal to the geometric mean of the minimum and maximum extent of each antenna temperature contour in a map. The maps fit are only different than those shown in Figures $1 a, 1 b$, and 2 in that they show $T_{A}$ contours at intervals of $0.1 \mathrm{~K}$ over the full $T_{A}$ range of each map. In maps that contain more than one closed contour for a given $T_{A}$, an "averaged $R$ " for that $T_{A}$ is used in the fits. The fits are not especially sensitive to this choice of fitting technique: the intrinsic scatter in the data has more of an effect on the fit than do measurement uncertainties for individual data points. Table 3 lists the results of the fits to antenna temperature as a function of contour extent for all of the maps shown in this paper and in Paper I.

\subsubsection{Line Width-Antenna Temperature Correlations}

Figures 6, 7a, and $8 a$ quantify the trend in the $\mathrm{OH}$ and $\mathrm{C}^{18} \mathrm{O}$ line width maps (Figs. $1 a, 1 b$, and 2 ): line width decreases systematically as antenna temperature increases. The parameters of linear fits to the $\log \Delta v_{\mathrm{NT}}-\log T_{A}$ data shown in Figures 6, 7, and 8 are given in Table 3. To convert from angular size to linear size, we assume that B1, TMC-1C, and L1251A are at distances of 300, 140, and 200 pc, respectively. The top axes used in Figures $6,7 a, 7 b$, and $8 a$ show the size scales corresponding to the temperatures shown on the bottom axes, as derived from the $T_{A}-R$ relations in $\S 4.2 .1$ (see Fig. 5) and listed in Table 3. Figures $7 b$ and $8 b$, which are reproduced from Paper I, show that the trend for line width to increase toward low antenna temperatures (large size scales) is significantly shallower within the cores themselves (as defined by $\mathrm{NH}_{3}$ emission) than in their environs (the $\mathrm{OH}$ or $\mathrm{C}^{18} \mathrm{O}$ emission). In fact, inside the half-power contour of the cores, line width is independent of size. This important change in the slope of the line widthsize relation is discussed as a "transition to coherence" in $\S 5.1 .1$.

To illustrate that the power-law fitting of line width as a function of antenna temperature is not especially sensitive to how one massages the data in advance of fitting, Figures $6,7 a$, and $8 a$ each demonstrate a slightly different method of data selection. In the B1 map (Fig. 6), which is the largest,

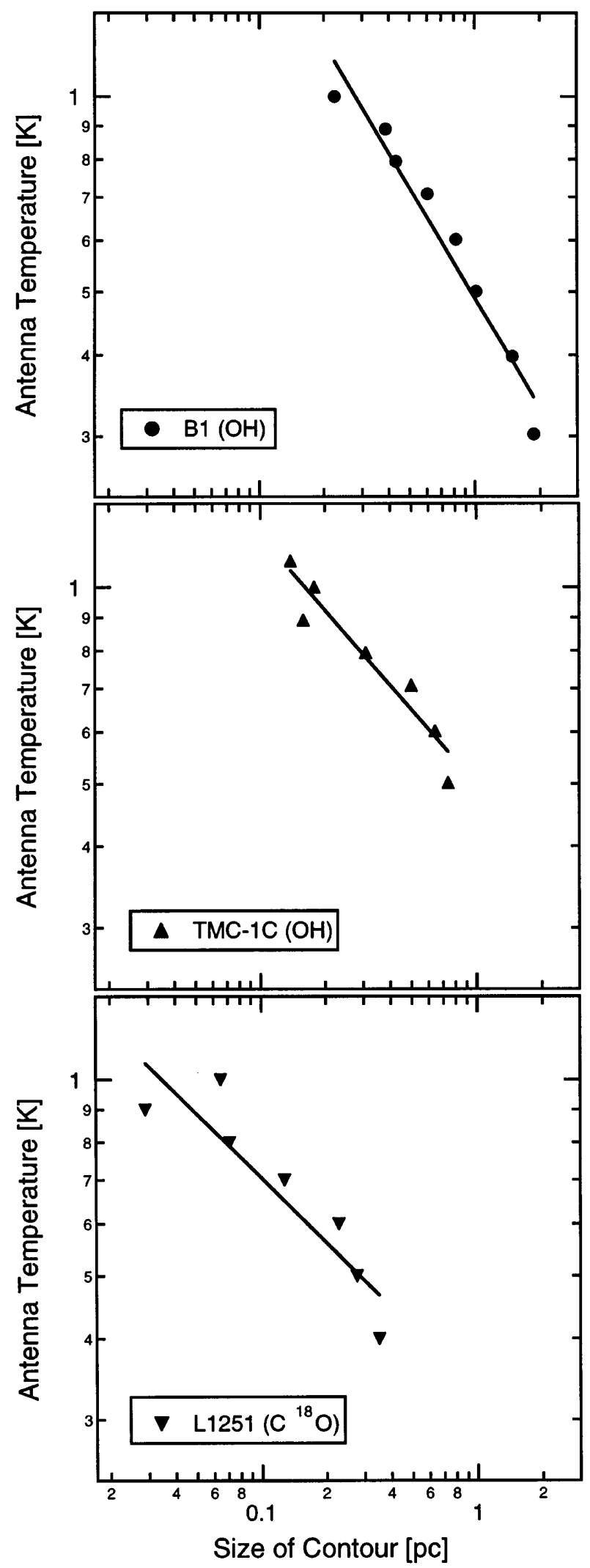

FIG. 5.-Antenna temperature as a function of radius. The three panels show relations between $T_{A}$ and $R$ for the maps of B1, TMC-1C, and L1251A shown in Figs. 1 and 2. In constructing these relations, each $T_{A}$ contour in a map is assigned a size equal to the geometric mean of the minimum and maximum extent of that contour. In maps that contain more than one closed contour for a given $T_{A}$, the average size of all the contours for that $T_{A}$ is plotted and used in fitting. The parameters of the power-law fits plotted in each panel are listed in Table 3. 


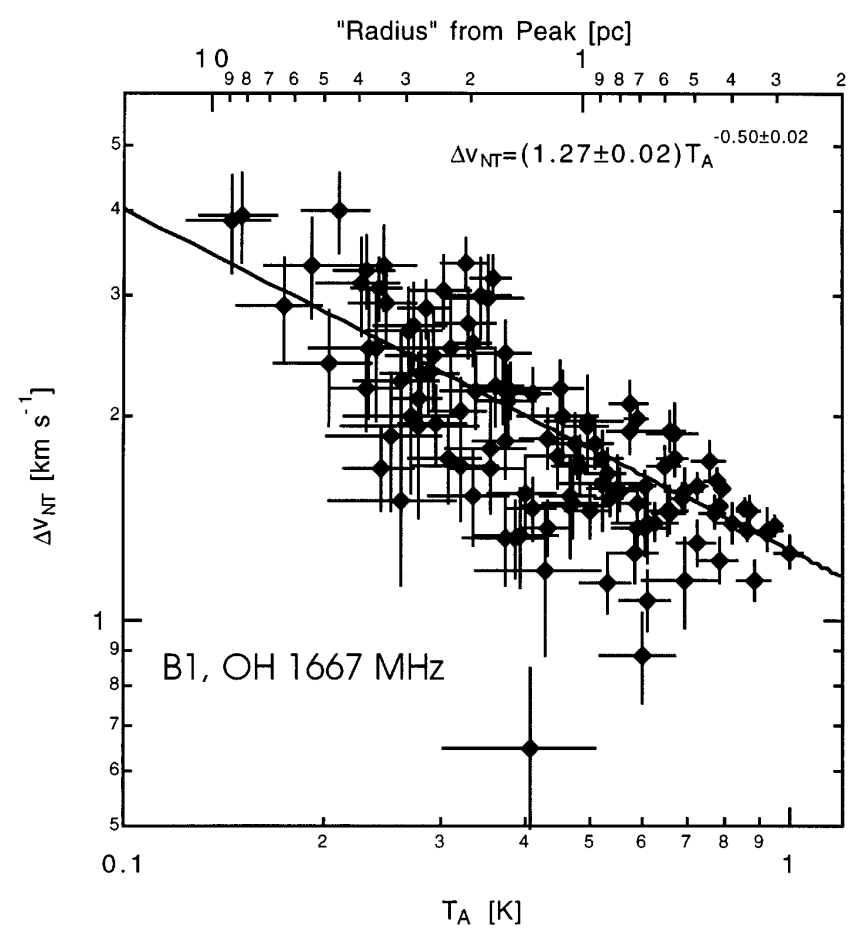

Fig. 6.- - Line width as a function of antenna temperature in B1. The $\mathrm{OH} 1667 \mathrm{MHz}$ spectra used to create this plot are also shown in map form in Fig. 1a. Antenna temperatures and line widths plotted are derived from single-component Gaussian fits. Each mapped position is shown as a single data point. The solid line shows the fit noted in the figure and listed in Table 3. The top axis shows the predicted size scale for the measured antenna temperature level, based on the $T_{A}-R$ fit in Table 3 . highest quality map in this paper, we show data points for every mapped position. For TMC-1C (Fig. 7a), which is of lower quality than the B1 map, only data points at which the Gaussian fits to the line profiles gave a signal-to-noise ratio greater than 4 are included. And, for the $\mathrm{C}^{18} \mathrm{O}$ data in L1251A (Fig. 8a), we have compressed the data by binning all points into $0.1 \mathrm{~K}$ bins (as was done for the $\mathrm{NH}_{3}$ data in Paper I and Figs. $7 b$ and $8 b$ ). In cases in which any of the three methods can be used (such as B1), all give the same fit results to within the quoted errors.

\subsubsection{Line Width-Size Correlations}

Combining the fitted $T_{A}(R)$ and $\Delta v_{\mathrm{NT}}\left(T_{A}\right)$ relations in Table 3 gives power-law expressions for $\Delta v_{\mathrm{NT}}(R)$, which are also listed in Table 3 . In the terminology similar to equation (1), where $\Delta v_{\mathrm{NT}} \propto R^{a}$, and noting that this is a Type 4 (single-tracer, single-cloud) relation, we typically find that $a_{4} \sim 0.2 \pm 0.06$ for the $n \sim 10^{3} \mathrm{~cm}^{-3}$ gas traced by $\mathrm{OH}$ and $\mathrm{C}^{18} \mathrm{O}$ and $a_{4} \sim 0.1 \pm 0.08$ for the full $\mathrm{NH}_{3}$ core maps. Considering the errors in these power-law slopes, one might not see them as significantly different. However, within the halfpower contours of the $\mathrm{NH}_{3}$ maps (the highest density portions of the cores), the slope of the line width-antenna temperature relation is typically consistent with zero (see Paper I). The change from line width increasing with size in a core's environs, but not within a core, implies that cores and their environs represent physically distinct regimes. This difference is only markedly apparent when comparing the interiors of cores (which have $a_{4} \sim 0$ ) with the core environments (see Figs. 9 and 10).
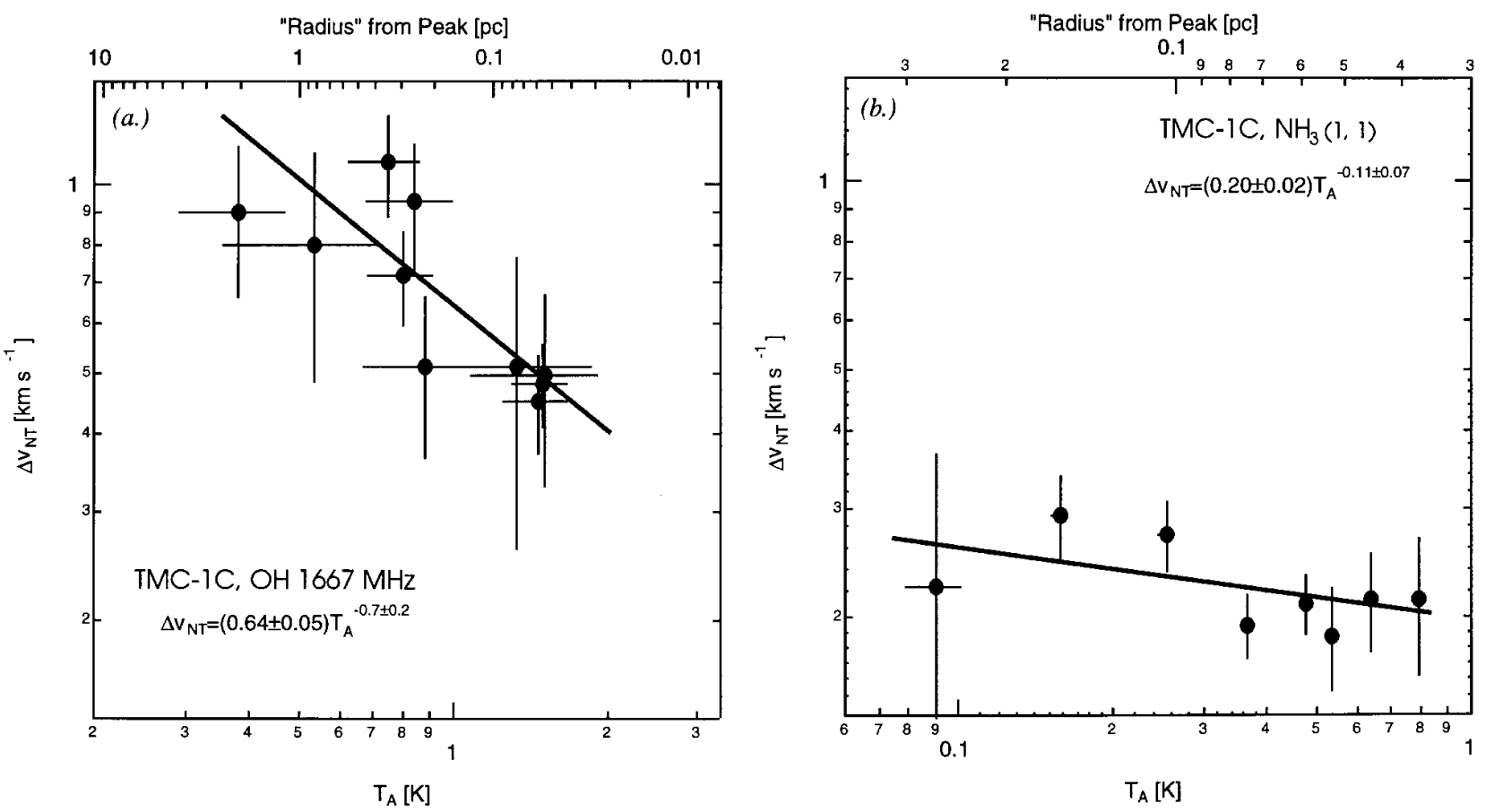

FIG. 7.-Line width as a function of antenna temperature in TMC-1C. Solid lines show the fits noted in the figures and listed in Table 3. Left: The OH $1667 \mathrm{MHz}$ spectra used to create this plot are also shown in map form in Fig. 1b. Antenna temperatures and line widths plotted are derived from single-component Gaussian fits. Each mapped position with adequate signal-to-noise ratio ( $\geq 4 \sigma$ in the fit) is shown as a single data point. Right: $\mathrm{NH}_{3}$ data from Paper I. Each data point represents the average of mapped positions that fall $\pm 0.05 \mathrm{~K}$ from the $T_{A}$ of the plotted point. (See Paper I for more information). Contrast the near independence of line width on antenna temperature in the $\mathrm{NH}_{3}$ data with the power law evident for the OH data in the left-hand panel. In both panels, the top axis shows the predicted size scale for the measured antenna temperature level, based on the $T_{A}-R$ fits in Table 3 . Note that the dynamic range in $T_{A}$ is equal in both panels. 

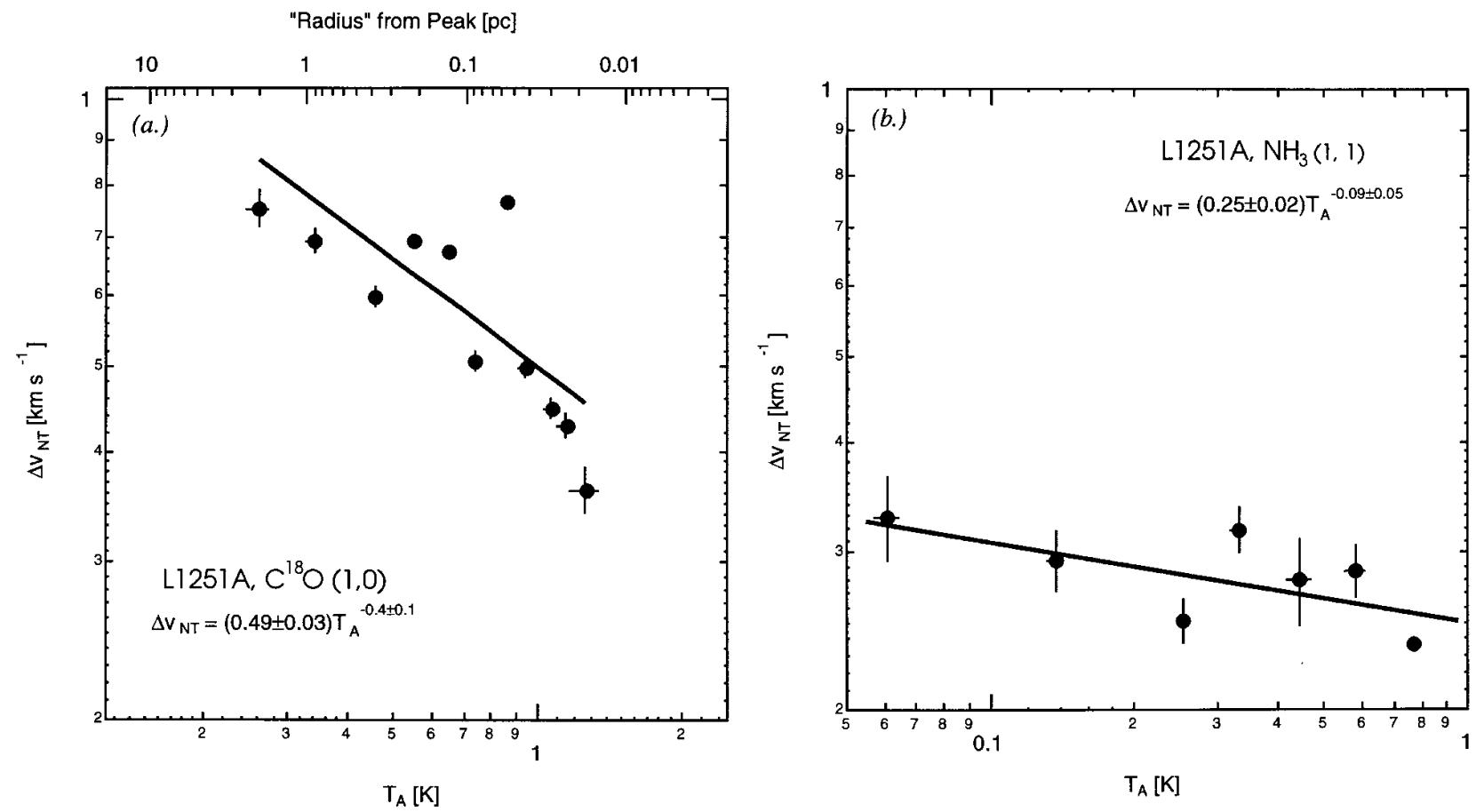

Fig. 8. - As in Fig. 7, but for L1251A. The $\mathrm{C}^{18} \mathrm{O}$ spectra used to create the plot on the left are also shown in map form in Fig. 2. In the left panel, each data point represents the average of mapped positions that fall $\pm 0.05 \mathrm{~K}$ from the $T_{A}$ of the plotted point, and the top axis shows the predicted size scale for the measured antenna temperature level, based on the $T_{A}-R$ fit in Table 3.

\subsubsection{Column Density-Size Relations}

The results of $\S \S 4.2 .1$ and 4.2 .3 can be used to estimate the radial dependence of column density, $N$, in cores and their surroundings. If, as in the case of low optical depth constant excitation temperature lines of sight, column density is proportional to $\int T_{A}(v) d v$, then in a map in which line profiles are well approximated by a single shape (e.g., a Gaussian),

$$
N \propto T_{A} \Delta v .
$$

So, if $T_{A} \propto R^{c}$ and $\Delta v \propto R^{a}$, then $N \propto R^{a+c}$. Using this simple approximation, Table 3 implies that for $\mathrm{OH}$ and $\mathrm{C}^{18} \mathrm{O}$ maps of core environments, $N \propto R^{-0.2}$, and for cores, as traced by $\mathrm{NH}_{3}, N \propto R^{-0.9}$. In other words, column density increases with decreasing size scale much faster in cores than it does in their environments. If cores have a much higher filling factor than the lower density gas around them, then this relation is explained naturally (see $\S 5.1$ and Fig. 10).

These column density profiles also imply that the dense gas traced by $\mathrm{NH}_{3}$ observations in the cores is closer to being in hydrostatic equilibrium than is the gas traced by the $\mathrm{OH}$ and $\mathrm{C}^{18} \mathrm{O}$ observations of the core surroundings. In hydrostatic equilibrium, gravitational pressure, which is proportional to $N R$, is balanced by kinetic pressure, which is proportional to $\Delta v^{2}$. Thus, for the $\mathrm{NH}_{3}$-traced gas, we find that gravitational energy is $\propto R^{0.1}$, and kinetic energy has a radial dependence that is quite similar, $\propto R^{0.2}$ (within errors of $\sim 0.1$ in the exponent). On the contrary, the radial dependences of these pressures for the $\mathrm{OH}-$ and $\mathrm{C}^{18} \mathrm{O}$ traced gas are quite different, even considering the errors, with gravitational pressure $\propto R^{0.8}$ and kinetic pressure $\propto R^{0.4}$. This result lends additional credibility to the idea

TABLE 3

Power-Law Relations among Line Width, Antenna Temperature, and Size

\begin{tabular}{|c|c|c|c|c|}
\hline Tracer & B1 & TMC-1C & L1251A & Characteristic Relation \\
\hline \multicolumn{5}{|c|}{ Antenna Temperature-Size } \\
\hline $\begin{array}{l}\mathrm{OH} \ldots \ldots \\
\mathrm{C}^{18} \mathrm{O} \ldots \ldots \\
\mathrm{NH}_{3} \ldots \ldots\end{array}$ & $T_{A}=(0.49 \pm 0.02) R^{-0.56 \pm 0.05}$ & $\begin{array}{l}T_{A}=(0.50 \pm 0.03) R^{-0.38 \pm 0.05} \\
T_{A}=(0.021 \pm 0.005) R^{-1.1 \pm 0.1}\end{array}$ & $\begin{array}{l}T_{A}=(0.33 \pm 0.05)^{-0.33 \pm 0.07} \\
T_{A} \propto R^{-1}\end{array}$ & $\begin{array}{l}T_{A} \propto R^{-0.5} \\
T_{A} \propto R^{-0.3} \\
T_{A} \propto R^{-1}\end{array}$ \\
\hline \multicolumn{5}{|c|}{ Line Width-Antenna Temperature } \\
\hline \multicolumn{5}{|c|}{ Line Width-Size } \\
\hline $\begin{array}{l}\mathrm{OH} \ldots \ldots \ldots \\
\mathrm{C}^{18} \mathrm{O} \ldots \ldots \\
\mathrm{NH}_{3} \ldots \ldots\end{array}$ & $\Delta v_{\mathrm{NT}}=(1.82 \pm 0.06) R^{0.28 \pm 0.03}$ & $\begin{array}{l}\Delta v_{\mathrm{NT}}=(1.0 \pm 0.2) R^{0.27 \pm 0.08} \\
\Delta v_{\mathrm{NT}}=(0.30 \pm 0.09) R^{0.12 \pm 0.08}\end{array}$ & $\begin{array}{l}\Delta v_{\mathrm{NT}}=(0.8 \pm 0.1) R^{0.13 \pm 0.04} \\
\Delta v_{\mathrm{NT}} \propto R^{0.09 \pm 0.05}\end{array}$ & $\begin{array}{l}\Delta v_{\mathrm{NT}} \propto R^{0.3} \\
\Delta v_{\mathrm{NT}} \propto R^{0.1} \\
\Delta v_{\mathrm{NT}} \propto R^{0.1}\end{array}$ \\
\hline
\end{tabular}




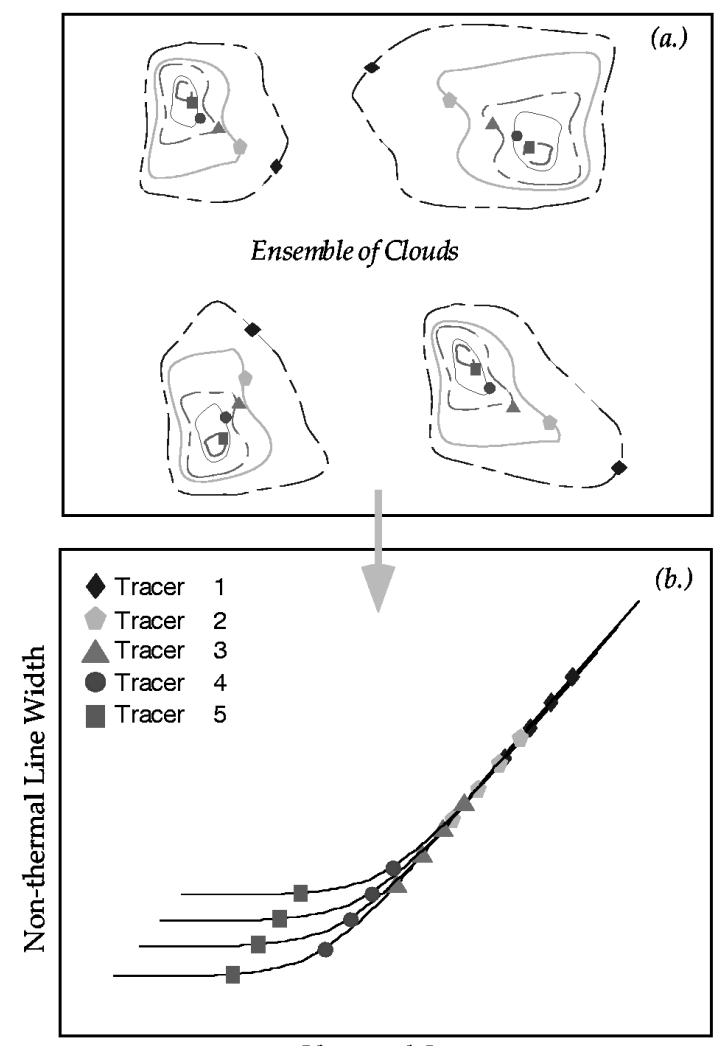

Observed Size

SiNGLE CLOUd OBSERVED IN MULTIPLE TRACERS

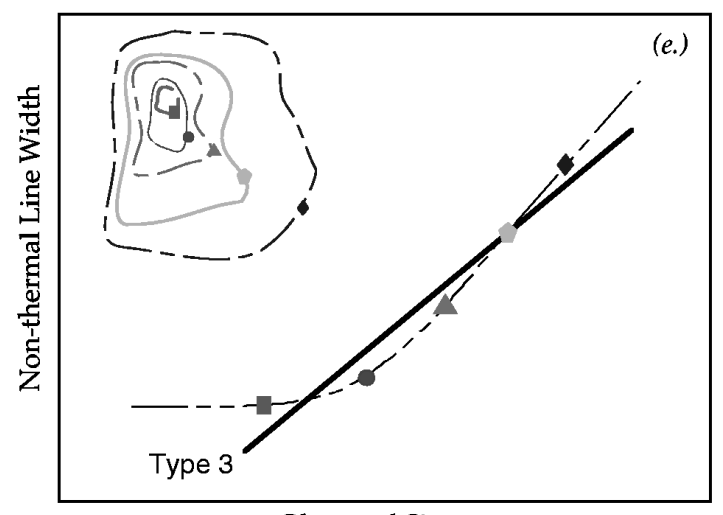

Observed Size
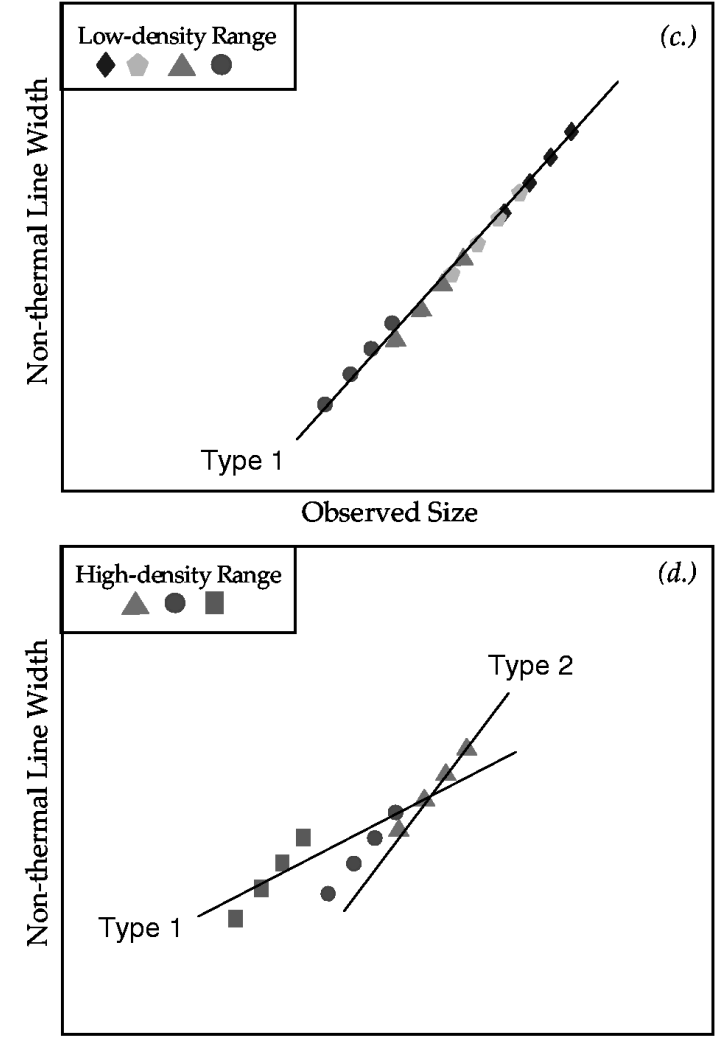

Observed Size

Single CLOUd OBSERVED IN SiNGLE TrACERS

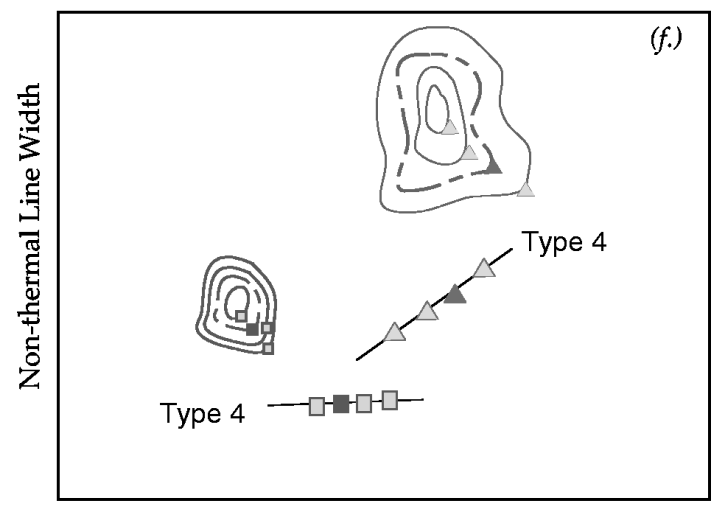

Observed Size

FIG. 9.- Types of line width-size relations. (a) Schematic diagram showing hypothetical half-power contours of five spectral lines probing systematically increasing density regimes in each of four regions. Symbols marking the contours are explained in the next panel. (b) For each contour shown in $(a)$, a corresponding point is plotted in the nonthermal line width-size plane. Solid curves show the (hypothesized) underlying relationship inherent in each region. These relationships are not easily identifiable from an ensemble of data or from sparsely sampled multitracer, single-cloud data, as in (e). Note that the larger size scale piece of the line width-size relation has been scaled, for graphic simplicity, so that all four clouds overlap. In reality, this is not at all likely be the case. (c) A subset of the points from $(b)$, which includes all points except those for the highest density tracer. This subset is representative of data that Larson (1981) included in his study that uncovered a Type 1 power-law relation between line width and size. $(d)$ A subset of the points in $(b)$, for the three highest density tracers. Without a priori knowledge of the underlying relationships shown in (b), the high-density ensemble can appear to give another Type 1 power law, this time with a slightly shallower slope than in $(c)$. The apparent cloud-to-cloud slope in the set of points for each tracer is produced by an assumption that each cloud is in virial equilibrium, so that a point at a fixed density moves to higher line width as the size scale over which it is found increases. Note that a subset of this data which compares multiple clouds in the same tracer constitutes an example of a Type 2 line width-size relation and that $a_{2}$ is not necessarily equal to $a_{1}$. Furthermore, a Type 3 relation using data from one cloud at a time (not shown in this panel; see [e]) would give yet another slope, $a_{3}$. $(e)$ Line width-size points from $(b)$ corresponding to a single cloud from the ensemble in $(a)$. The heavy solid line indicates a Type 3 power-law fitted to all of the data points. Without nearly "perfect" data over this density-size-line width range, it is nearly impossible to identify an underlying relationship like the one shown by the broken line. $(f)$ This panel puts the contribution of this paper and Paper I into context. The data points shown are not a subset of the data from $(b)$. Instead, they represent the observed dependence of line width on size for a single cloud, studied in one tracer at a time- a Type 4 line width-size relation. The squares in the left-hand side of the panel are for a coherent core, in which line width is observed to be virtually independent of size. The triangles toward the upper right show how line width measurements vary with size for the larger region around the core. The solid square and solid triangle represent the FWHM value for the two tracers, which would be used in a Type 1 or Type 3 study. 


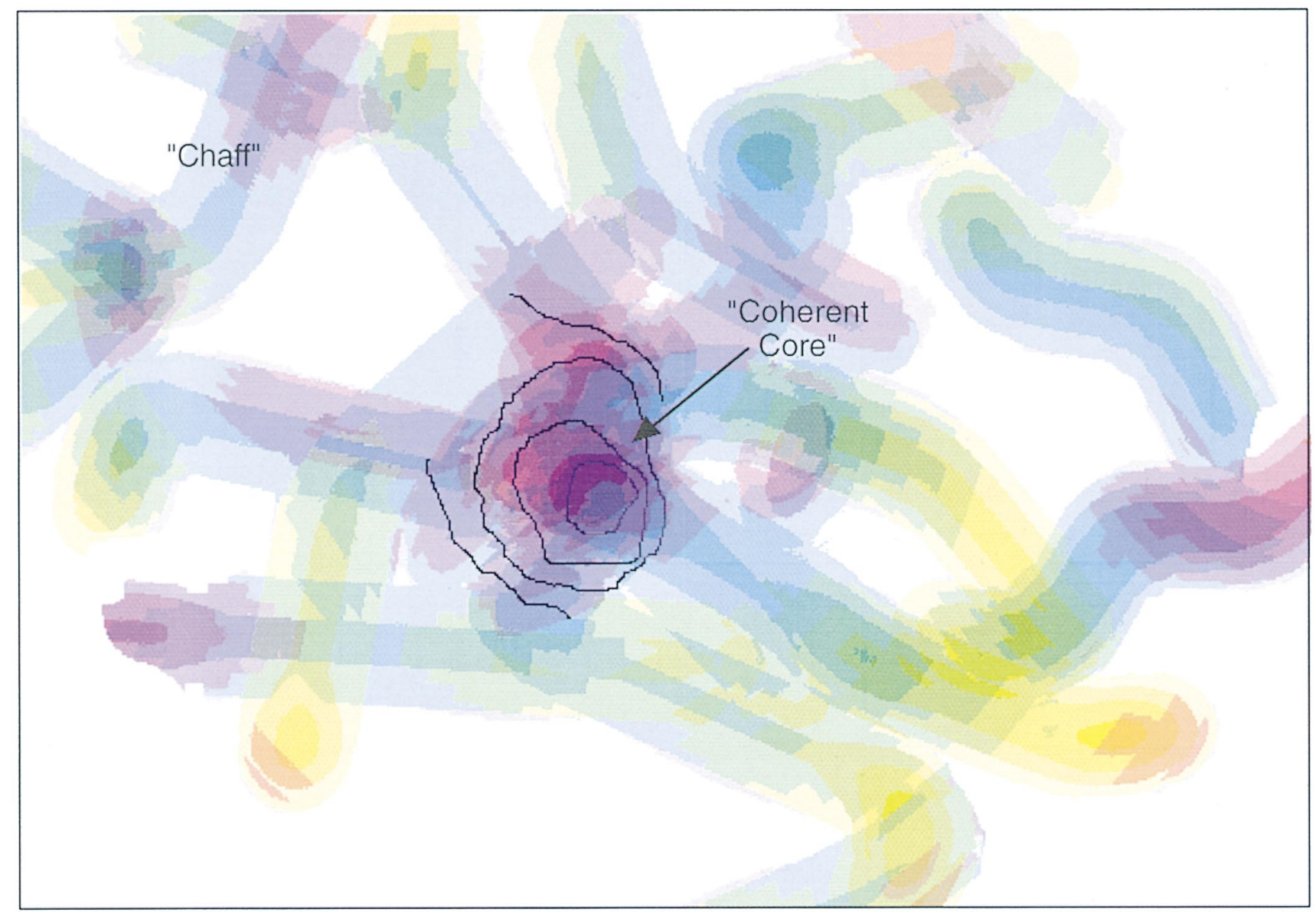

FIG. 10.-An illustration of the transition to coherence. Color and shading schematically represent velocity and density in this figure. On large scales, material (labeled chaff) is distributed in a self-similar fashion, and its filling factor is low. On scales smaller than some fiducial radius, the filling factor of gas increases substantially, and a coherent dense core, which is not self-similar, is formed. Due to limitations in the authors' drawing ability, the figure emphasizes a particular size scale in the chaff, which should actually exhibit self-similar structure on all scales ranging from the size of an entire molecular cloud complex down to a coherent core.

that a "coherent core" is close to being a high filling factor blob of gas in some kind of equilibrium, while the environment of that core is of a more temporary and turbulent nature.

\section{DISCUSSION}

\subsection{A Self-Consistent View of Cores and their Environs}

For the purposes of discussion, consider a region like the one pictured in Figure 10. On large scales, what is often called a "cloud" consists of gas we label "chaff". The chaff has a low filling factor and is presumed to be distributed in a self-similar way, even though the illustration emphasizes a particular scale. This chaff is likely comprised of material whose kinematics are dominated by turbulence and whose distribution is self-similar (see Elmegreen \& Falgarone 1996, and references therein). The chaff, having self-similar structure, is not taken to represent a uniformly low-density "intercloud" medium. There is dense gas in the chaff, just not very much of it. ${ }^{8}$ In some small region immersed in the chaff, the filling factor of the gas increases substantially and the gas settles down to a single velocity dispersion. We will call this small region of high filling factor and roughly constant velocity dispersion a coherent core.

\footnotetext{
${ }^{8}$ This hypothesis is supported by recent observations of $\mathrm{CO}$ isotopes in the Rosette molecular complex (Schneider et al. 1996).
}

This situation can arise if the outer scale of the coherent core $R_{\text {coh }}$ is taken to represent a boundary between physical regimes. In applying this cartoon to the conditions in a real molecular cloud, $R_{\text {coh }}$ can be incorporated into several relevant theoretical pictures (see $\S 5.3$ ). Since the nonthermal line width associated with $R_{\text {coh }}$ is usually close to the thermal line width, we might expect that $R_{\text {coh }}$ is related to $R_{\mathrm{TNT}}$, the radius at which thermal and nonthermal velocity dispersion are exactly equal (Myers \& Fuller 1992; see also Table 3). More potential transition points become relevant when magnetic fields are explicitly included in the picture. For the static field, ambipolar diffusion will reduce the ability of magnetic forces to support gas against collapse when the ionization fraction drops below a critical value. And, for the varying field, poor ion-neutral coupling causes a minimum size scale cutoff in the spectrum of Alfvén waves which permeate the ISM (e.g., Arons \& Max 1975; Zweibel \& Josafatsson 1983; Carlberg \& Pudritz 1991). We will return to discussion of these possible physical transitions in $\S 5.3$, after we have demonstrated how this picture of a transition to coherence is empirically justified by our data.

Inside the core, the gas is not necessarily as featureless as an isothermal sphere. The "coherent blob" is still subject to fragmentation by instabilities, and it can easily harbor forming stars with accompanying circumstellar disks and envelopes. 


\subsubsection{The Transition to Velocity "Coherence"}

The connection between the hypothetical view in Figure 10 and our spectral line maps is easy to make. On larger scales, the $\mathrm{C}^{18} \mathrm{O}$ and $\mathrm{OH}$ data show line width increasing with size in a positive-index power-law fashion, which is indicative of a self-similar or turbulent medium. On the smaller scales traced by $\mathrm{NH}_{3}$, there is almost no dependence of line width on size. What little increase in line width there is appears to come only from a modest rise in line width at the core edges. Inside the half-power contour of a core, we typically see a uniform distribution of line width, which is indicative of the dispersion in a coherent dense core (see Paper I). The size of a typical half-power $\mathrm{NH}_{3}$ contour is $\sim 0.1-0.2 \mathrm{pc}$, which we will take as a good estimate of $R_{\text {coh }}$.

If this nearly constant line width in the core were equal to the thermal line width of tracer, this result would be easier to understand. However, the velocity dispersion at which the cores become coherent is always significantly greater than the thermal velocity dispersion (for the tracer molecule, $\mathrm{NH}_{3}$ ) but less than the thermal line width of $\mathrm{H}_{2}$. In other words, the turbulence remaining in the coherent cores is always observed to be subsonic. For the four cores featured in Paper I, the mean $\mathrm{NH}_{3}$ intrinsic FWHM line width is $\Delta \bar{v}_{\text {int }}=0.28 \mathrm{~km} \mathrm{~s}^{-1}$. At a temperature of $10 \mathrm{~K}$, the thermal FWHM for $\mathrm{NH}_{3}$ is $0.17 \mathrm{~km} \mathrm{~s}^{-1}$ and the thermal FWHM for an $\mathrm{H}_{2}$ molecule is $0.47 \mathrm{~km} \mathrm{~s}^{-1}$. Thus, the nonthermal component of the line width in a typical coherent core is

$$
\begin{aligned}
\Delta v_{\mathrm{NT}} & =\left[\Delta \bar{v}_{\mathrm{int}}^{2}-\Delta v_{T}\left(\mathrm{NH}_{3}\right)^{2}\right]^{1 / 2} \\
& =\left(0.28^{2}-0.17^{2}\right)^{1 / 2}=0.22 \mathrm{~km} \mathrm{~s}^{-1} .
\end{aligned}
$$

So, for an $\mathrm{H}_{2}$ molecule in a typical coherent dense core, the total line width would be

$$
\begin{aligned}
\Delta v & =\left[\Delta v_{\mathrm{NT}}^{2}+\Delta v_{T}\left(\mathrm{H}_{2}\right)^{2}\right]^{1 / 2} \\
& =\left(0.22^{2}+0.47^{2}\right)^{1 / 2}=0.52 \mathrm{~km} \mathrm{~s}^{-1}
\end{aligned}
$$

which is barely, but measurably, larger than the thermal $\mathrm{H}_{2}$ width $\left(0.47 \mathrm{~km} \mathrm{~s}^{-1}\right)$ alone. Small changes in the kinetic temperature do not have a large effect, since the thermal line width increases only as the square root of the temperature. In order to account for the typical $\Delta \bar{v}_{\text {int }}$ as a purely thermal line width (i.e., $\Delta v_{\mathrm{NT}}=0$ in eq. [6]), the core temperature would need to be $29 \mathrm{~K}$. Any temperature greater than about $15 \mathrm{~K}$ is inconsistent with measurements of the temperature of low-mass dense cores based on $\mathrm{NH}_{3}$ line excitation (Benson \& Myers 1989). Thus, a "coherent dense core" is very similar, but not identical to, a thermal ball of gas.

\subsubsection{Comparison with Stellar Clustering Scales}

The size scale, $R_{\text {coh }} \sim 0.1 \mathrm{pc}$, characterizing the transition in the line width-size scaling relationship is just a few times larger than the size scale ( $\sim 0.04 \mathrm{pc})$ at which Larson (1995) identifies a break in the power-law slope of the function describing the clustering of newly formed stars in the Taurus dark cloud complex (Fig. 11). The possibility of a break in slope for the stellar clustering function in Taurus was first noted by Gomez et al. (1993). Larson's study added additional data from the literature that made the break more evident. The size scale of the break in the starclustering power law has been likened to a Jeans size by Larson (see $\S$ 5.3.4). We expect that our result and Larson's are similar, in that we are finding approximately Jeans mass clumps with roughly constant line width, and he is studying the fruits of those clumps. Figure 11 illustrates this similarity by plotting both stellar clustering and line width as functions of size. ${ }^{9}$

Consider for a moment how the distribution of gas and stars will be related on scales much larger than a coherent core. After stars form, the stars themselves are subject only to gravitational forces, while the remaining gas is subject to gravitational, pressure gradient, and magnetic forces. Over time, this distinction can make it increasingly difficult to compare the stellar and gas distributions directly. However, if gravitational forces dominate, or the elapsed time since star formation is not too long, then the properties of the stellar distribution on large scales $(\gg 0.1 \mathrm{pc})$ in molecular clouds like Taurus should match the gas distribution properties. In other words, the stars will be found wherever there happened to be gas to form them. For example, if the gas distribution is fractal, the stellar distribution should be fractal, with a similar fractal dimension (i.e., Elmegreen \& Falgarone 1996). In massive star-forming regions, there is evidence that the gas and stellar distributions are related, since both gas and stars "cluster" together into large associations (Lada 1992), but the detailed correspondence between the stellar and gas distributions is not yet well determined.

On scales smaller than a coherent core $(<0.1 \mathrm{pc})$, the spatial distribution of stars is not likely to be directly related to the gas distribution. Instead, we expect that the fragmentation of coherent blobs, along with the action of outflow(s) and tidal forces, will ultimately determine the stellar distribution.

In this picture, the large-scale distribution of stars in Figure 11 is determined by the overall large-scale distribution of gas, while the small-scale distribution results from the fragmentation of coherent cores (see also Larson 1995).

\subsection{The Meaning of Line Width-Size Relations}

Figure 9 is not just a cartoon. Line width-size observations can truly give four different slopes $\left(a_{1}, a_{2}, a_{3}\right.$, or $\left.a_{4}\right)$, depending on how they are constructed. Figure $12 a$ shows all of the derived Type 4 line width-size relations tabulated in Table 3, plotted for the size range from the minimum to the maximum "radius" measured in the data set that produced each power law. In the remaining three panels of Figure 12, the other three Types of line width-size relations are illustrated for the same data set (see figure legend). The figure clearly shows that the slopes of the Type 1-4 relations need not be, and are not, identical. In fact, much can be learned from contemplating the difference between Types of line width-size relationships.

In order to understand the meaning of each Type, one must consider the selection effects inherent in spectral line observations of a given tracer. In practice, each tracer is sensitive to a relatively narrow range of density. Often, a critical density, above which a particular molecular energy level is sufficiently populated by collisions, is quoted as the minimum density traced in an observation, but little is ever said about the maximum density traced by a given spectral line probe. This maximum is affected by the spatial and velocity distribution (optical depth) of the material, as well as by chemical effects that cause particular tracers to exist

\footnotetext{
9 The nonthermal line width-size relation shown in Figure 11 uses equation (9) with $\sigma_{0}=0.13 \mathrm{~km} \mathrm{~s}^{-1}, R_{0}=0.2 \mathrm{pc}$, and $a=0.5$, which are the means of the parameter ranges used in the simulations presented in $\S 5.2 .1$ that reproduce Larson's Laws.
} 


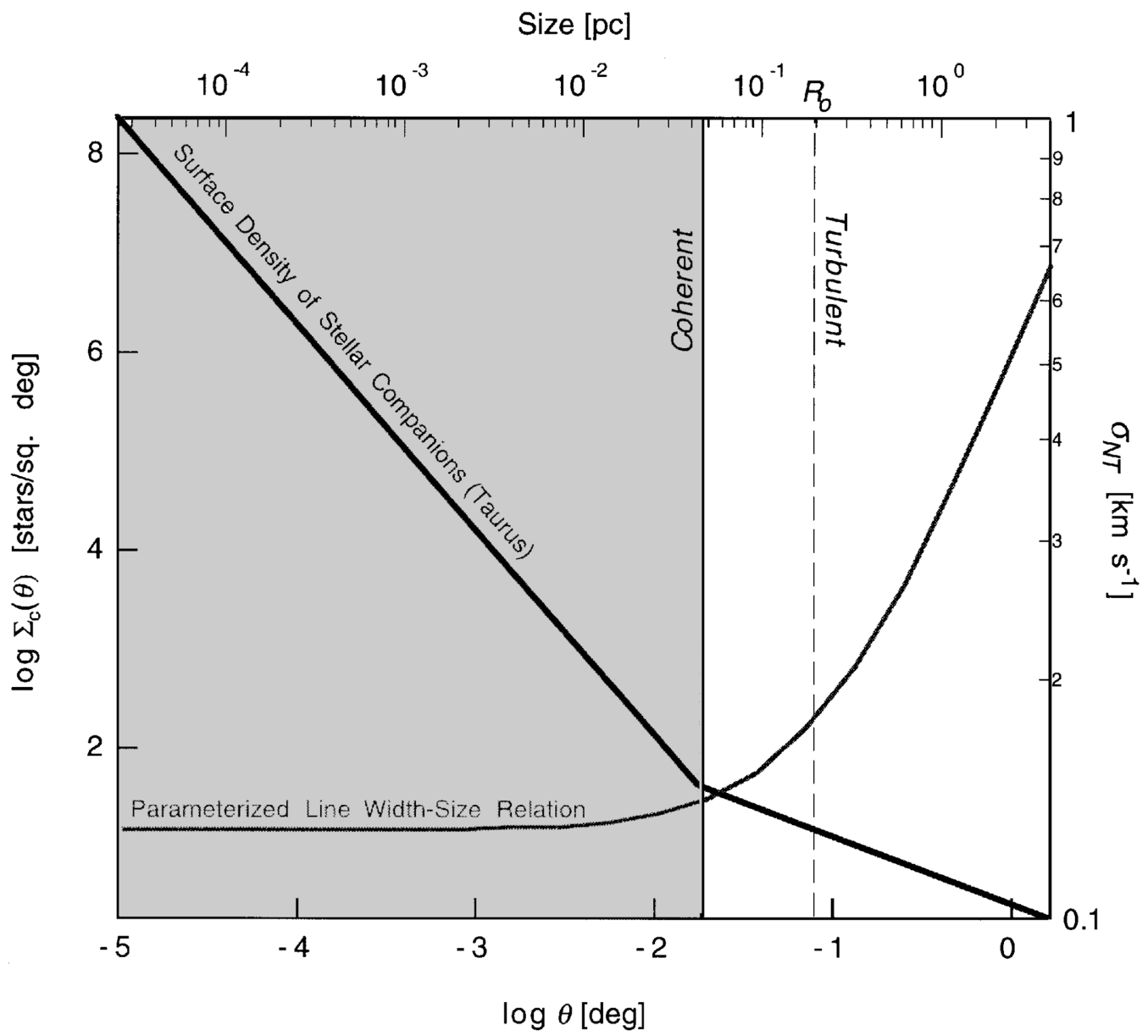

Fig. 11.-Comparison of stellar clustering properties with a line width-size relation. The relation between the surface density of stellar companions and angular separation is reproduced from Larson (1995). The surface density is plotted on the left axis vs. angular separation on the bottom axis. The top axis shows the linear sizes corresponding to the angular sizes on the bottom axis, at the distance of Taurus (140 pc), which is home to all of the stars in Larson's study. The parameterized line width size relation (right-hand axis) shows eq. (9) for the nonthermal velocity dispersion as a function of size, with $\sigma_{0}=0.13 \mathrm{~km}$ $\mathrm{s}^{-1}, R_{0}=0.2 \mathrm{pc}$, and $a=0.5$, using the top axis for $R$. Notice that both the gas distribution and the stellar distribution go through transitions in a range of sizes near $0.1 \mathrm{pc}$.

only under a limited range of conditions (Kuiper, Langer, \& Velusamy 1996). Hence, the dynamic range of a tracer used to study the distribution of gas is itself a function of the gas distribution and chemistry. Deriving this maximum density analytically is quite a challenge, but, happily, in cases where multitransition observations of a single tracer (which allow for direct estimates of volume density) are available, density can be directly measured as a function of map position. The maximum dynamic range in the density traced by a single probe in a single map is almost always less than 2 orders of magnitude and often less than a factor of 10 (Scalo 1987; Bergin, Snell, \& Goldsmith 1996). Suffice it to say here that in typical molecular clouds, each tracer used is sensitive to at most a factor of 2 orders of magnitude in density.

So, given that each tracer essentially represents a density regime, observations of Type 3 , in which multiple tracers are compared for a single region, give the best idea of the overall density profile in a region. Indeed, Type 3 relations have been shown to match hydrostatic equilibrium models of star-forming clouds (Fuller \& Myers 1992). Single-tracer, single-cloud (Type 4) line width-size relations are a diagnostic of the distribution of gas within a density regime and are probably the best diagnostic of the dynamical processes applicable within that regime. For example, we think that the Type 4 correlations in Table 3 listed for $\mathrm{OH}$ or $\mathrm{C}^{18} \mathrm{O}$ are representative of a turbulent or wave-permeated medium, whereas those listed for $\mathrm{NH}_{3}$ are relevant to the interior of an almost-thermal quiescent blob of gas.

Comparison of Type 1 (multitracer, multicloud) observations with Type 3 (multitracer, single-cloud) observations gives an estimate of the range of Type 3 relations present in different regions of the ISM. Previous studies (Fuller \& Myers 1992; Caselli \& Myers 1995) have found $0.2<a_{3}<$ 0.7. Regions of massive star formation tend to have smaller values of $a_{3}$ than do regions of low-mass star formation (Caselli \& Myers 1995), but no predictive model has been made of the detailed variation in slope from one region to another. Some (but not all) of the variation in Type 3 slopes is due to variations in the opacity and chemistry of the regions studied, since a FWHM contour for a particular tracer does not always represent exactly the same set of physical conditions (e.g., the same density). ${ }^{10}$ Also, if a Type 3 relation is only sparsely sampled and then fit with a single power law (e.g., a straight line is fit to three data points in a

\footnotetext{
${ }^{10}$ For example, even though the CS $(2-1)$ transition has a critical density greater than $10^{5}$, which is almost 2 orders of magnitude higher than the $(J, K)=(1,1)$ transition of $\mathrm{NH}_{3}, \mathrm{CS}(2-1)$ maps often trace larger regions with larger line widths than $\mathrm{NH}_{3}$ maps (e.g., Zhou et al. 1989).
} 


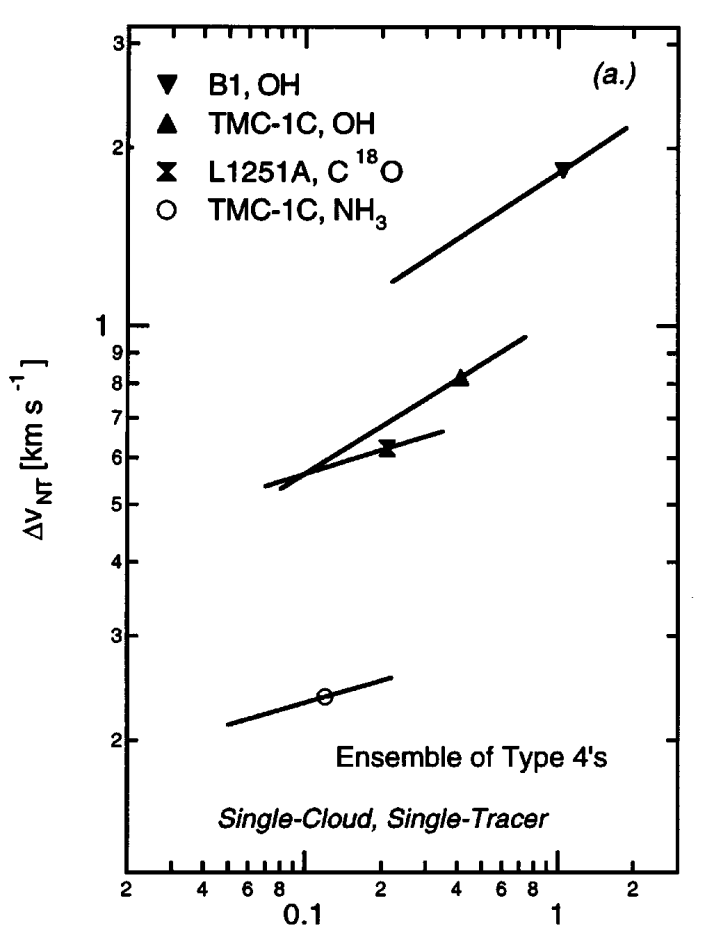

$\mathrm{R}[\mathrm{pc}]$

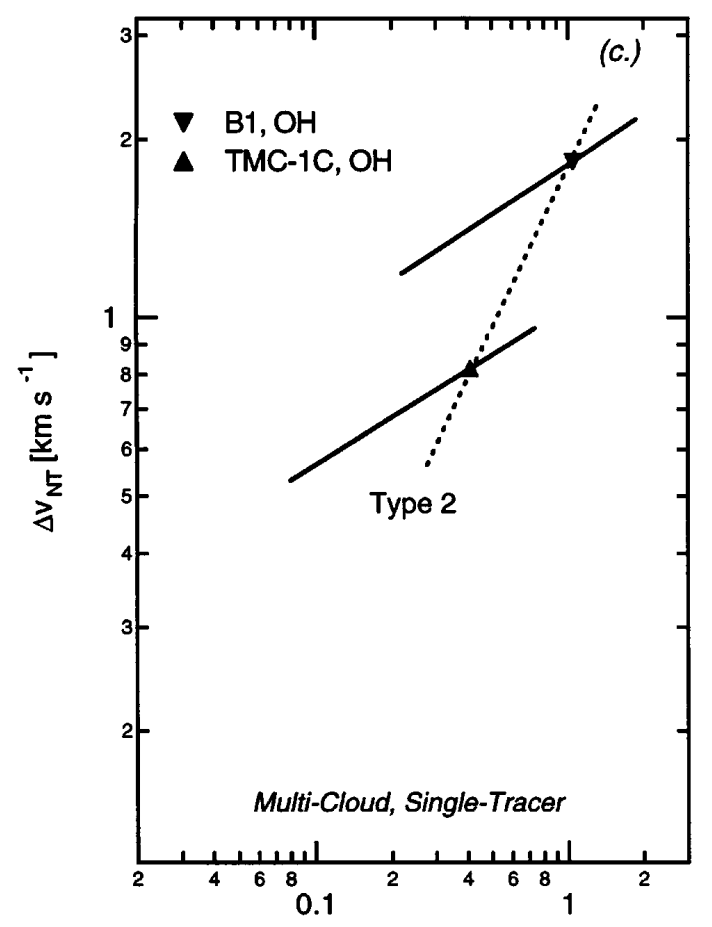

$\mathrm{R}[\mathrm{pc}]$
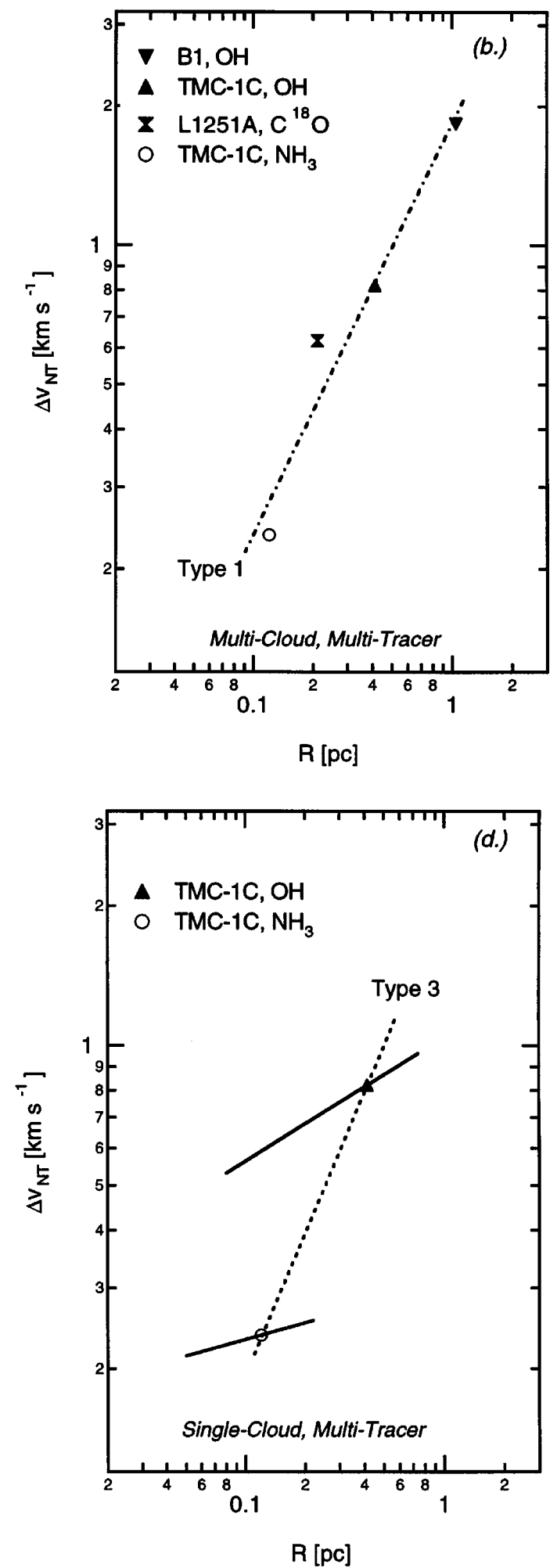

Fig. 12.-Four Types of line width size relation derived from the same data set. In (a), short line segments show the derived Type 4 line width-size relations tabulated in Table 3, plotted for the size range from the minimum to the maximum radius measured in the data set that produced each power law. The symbol associated with each line segment is plotted at the FWHM radius of the tracer it labels. In $(b)$, only the FWHM points from $(a)$ are shown, and a line width-size relation of Type 1 is drawn. In (c), only FWHM points observed with $\mathrm{OH}$ as the tracer are shown, which gives a (very sparsely sampled) Type 3 relation. In $(d)$, only data points for the TMC-1C cloud are plotted, yielding a (very sparsely sampled) Type 2 line width-size relation. Notice that the slopes of the Types 1-4 relations need not be, and are not, equal.

$\log \Delta v$-log $R$ plot), the slope derived will depend heavily on how close the set of tracers use is to the "transition" zone shown as a bend in the dashed curve in Figure 9e. In $\S 5.2 .1$, we show how mixing together a realistic sample of Type 3 relations can produce the Type 1 correlations quoted in the literature (e.g., Larson's Laws).

The meaning of Type 2 (single-tracer, multicloud) correlations is less obvious. Consider Figure 10 and a hypotheti- 
cal tracer $x$, which is only sensitive to densities within a factor of 10 of $n_{x}$. If $n_{x}$ is small relative to the typical density at which coherent cores are found (i.e., $n_{x} \ll 10^{4}$ ), then tracer $x$ will be distributed in a self-similar fashion wherever the density is near $n_{x}$, throughout a large volume and can, in effect, trace many size scales. The overall extent of tracer $x$, and similarly of all other tracers up to those characterizing the coherent (non-self-similar) cores, will appear to increase with the overall extent of the chaff shown in Figure 10. Thus, depending on how it is sampled, emission from tracer $x$ can represent a variety of size scales.

Scalo (1987) has pointed out that the range of column density traced by a particular set of spectral line observations is limited by the sensitivity of the observing system to a dynamic range of $\sim 10$. This is perhaps a conservative estimate, but it does imply that if an object is centrally condensed at some peak and extended over a large volume, then lines of sight farther from the peak will sample lower average density and thus need longer path length to give the same (detectable) column density. This effect is not necessarily problematic and can in fact be used as a method for relating plane-of-the-sky and line-of-sight size scales (see Fig. 4 and $\S 4.2 .1$ ). The potential problem with Type 2 relations, though, is that when the distance to clouds varies substantially, different size scales and volume densities will characterize the detectable regimes at the different distances, as illustrated in Figure 13. Scalo argues that if the column density traced is constant, then $n \propto 1 / R$ automatically, which gives $a_{2}=0.5$ if clouds are close to virial equilibrium (see eqs. [1], [2], and [3]). There is some experimental justification for this hypothesis. ${ }^{11}$ Solomon et al. (1987) find that $a_{2}=0.5$ for a sample of 273 molecular clouds observed in $\mathrm{CO}$, and they conclude that the clouds are near virial equilibrium and that the $\mathrm{CO}$ emission arises in a region of nearly constant mean surface density (i.e., in particular density layers within the chaff). Heyer, Carpenter, $\&$ Ladd (1996) also find $n \propto R^{-1}$ in a large CO data set, and they conclude that most of the emission comes from extended low column density material, globally characterized by the $A_{\mathrm{V}} \sim 0.5-1$ needed to form molecules.

Using a principal components analysis on four large $\mathrm{CO}$ data sets from Heyer et al. (1996), Heyer \& Schloerb (1996) find slopes for Type 4 line width size relations of about 0.5. ${ }^{12}$ The ${ }^{12} \mathrm{CO}$ and ${ }^{13} \mathrm{CO}$ observations used in the Heyer \& Schloerb study probe densities 1-2 orders of magnitude lower than those traced by $\mathrm{C}^{18} \mathrm{O}$ or $\mathrm{OH}$, and the regions studied were not specifically selected to be associated with low-mass dense cores. Nonetheless, when looking at Figure $12 a$ and noting that $a_{4} \approx 0.5$ for regions with density $\sim 100$ $\mathrm{cm}^{-3}$ (and size approximately tens of pc), it is tempting to speculate that $a_{4}$ increases progressively as lower densities are probed and that $a_{4}=0.5$ is as steep as it gets. If column density is constant, $a_{4}>0.5$ implies a nonequilibrium situation in which clouds are overpressured and unbound. Such clouds would presumably evolve toward a condition in which $a_{4} \leq 0.5$.

\footnotetext{
${ }^{11}$ Recent numerical work by Vázquez-Semadeni, Ballesteros-Paredes, \& Rodriguez (1997) also supports this hypothesis.

${ }_{12}$ Heyer \& Schloerb interpret these relations as Type 4 because the single-tracer data were analyzed region by region. Others might think of these as Type 2 (single-tracer, multicloud) relations (e.g., similar to the Solomon et al. 1987 and other large multiregion CO surveys), but the authors of the study find the Type 4 designation more appropriate (M. H. Heyer \& F. P. Schloerb 1997, private communication).
}

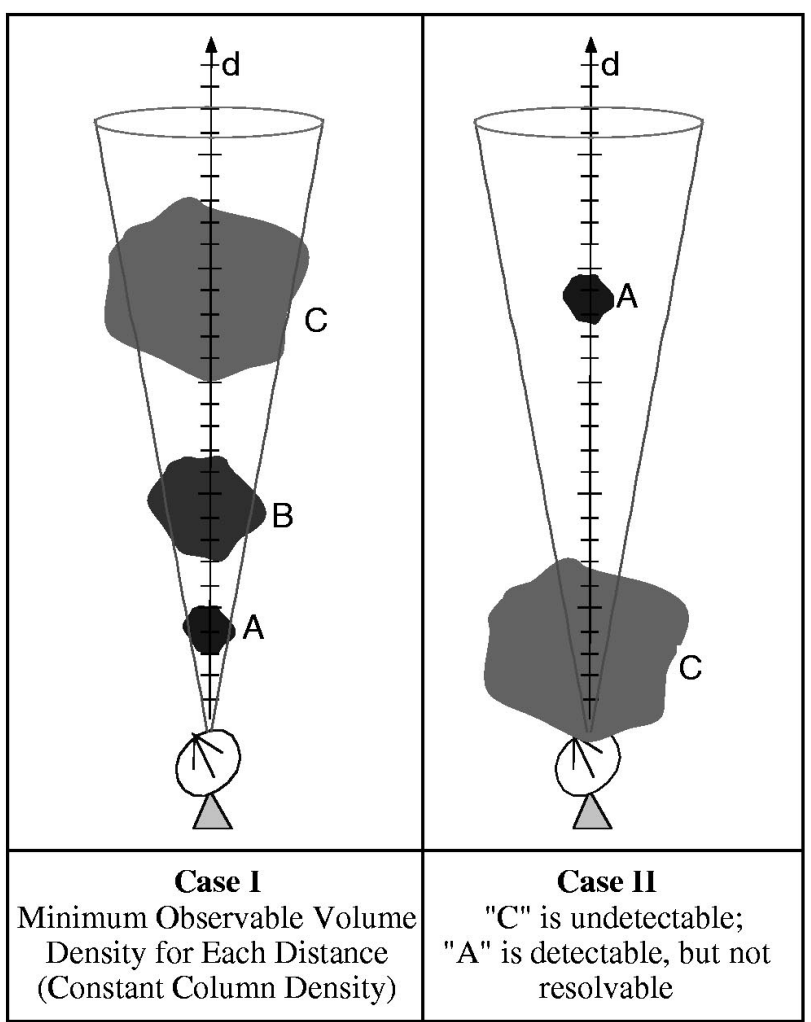

FIG. 13.- Selection effect in Type 2 line width-size relations. In Case I, the volume density $n$ of each cloud shown decreases linearly with distance $d$ from the observer. Hence, the column density $N \propto n d$ is the same for each cloud. Since beam area increases as $d^{2}$ and surface brightness $(\propto N)$ decreases as $d^{-2}$, the detectability of each cloud shown is the same. In Case II, we show that if cloud C is moved to where cloud A was in Case I, it would become undetectable because the column intersected by the beam is too small; and if cloud A is moved to the Case I distance of $\mathrm{C}$, it is detectable, but unresolved.

\subsubsection{Parameterizing Realistic Conditions}

Observed velocity dispersion is often decomposed into a thermal and nonthermal part using the formalism from equation (4):

$$
\sigma_{\mathrm{obs}}^{2}=\sigma_{T}^{2}+\sigma_{\mathrm{NT}}^{2}
$$

(Myers 1983). Since our observations seem to indicate that $\sigma_{\mathrm{NT}}$ approaches a constant value at small radii which is not strictly zero, we write the nonthermal line width as

$$
\sigma_{\mathrm{NT}}^{2}=\sigma_{0}^{2}\left[1+\left(R / R_{0}\right)^{2 a}\right] .
$$

This parameterization allows $\sigma_{\mathrm{NT}}$ to become asymptotic to a value $\sigma_{0}$ at a fiducial radius $R_{0}$. In the limit that nonthermal line width actually goes to exactly zero $\left(\sigma_{0} \rightarrow 0\right)$, equation (9) reduces to

$$
\sigma_{\mathrm{NT}}=\left(\frac{\sigma_{0}}{R_{0}^{a}}\right) R^{a},
$$

which is just another form of equation (1). ${ }^{13}$ This limit also holds as $R \rightarrow \infty$.

\footnotetext{
${ }^{13}$ Notice that in this parameterization, which was specifically chosen so that eq. (10) would give a line width-size relation like the one used by Myers and collaborators, $\sigma_{\mathrm{NT}}\left(R_{0}\right)=(2)^{1 / 2} \sigma_{0}$.
} 
The functional form for nonthermal line width given in equation (9) is used to draw the underlying relations shown as curved lines in Figure 9 and the line width-size curve in Figure 11. Our hypothesis is that a very densely sampled set of multitracer, single-cloud (Type 3) observations extending to small radii and high densities would actually reveal this relation. This parameterization is consistent with both our conceptual picture of coherence, where $\sigma_{0}$ is indicative of the velocity dispersion in the coherent region, and with the various Types of line width-size analyses. It is interesting to note that a Type 2 (single-tracer, multicloud) study will still see a positive power-law slope in the line width-size relation, even at very small radii, due to the fact that a given tracer is only sensitive to a narrow range of densities (see Fig. $9 d$ and Fig. 1 of Myers \& Fuller 1992 for schematic and real examples of this effect, respectively). The apparent cloud-to-cloud slope in the Type 2 relation results if each cloud is in approximate virial equilibrium, so that a point at a fixed density moves to higher line width as the size scale over which it is found increases.

In order to test how closely this parameterization matches available data, we created a synthesized Type 1 line width-size relation by mixing together a number of Type 3 relations created using a realistic range of parameters in equation (9). Specifically, we evenly filled parameter space with simulations of Type 3 relations (eq. [9] with $a=a_{3}$ ) in which $0.4<a<0.6,0.08<\sigma_{0}<0.18 \mathrm{~km} \mathrm{~s}^{-1}$, and $0.1<$ $R_{0}<0.3 \mathrm{pc}$. The range of values for each parameter was selected as representative of the Type 4 studies presented in this paper. Using these simulations, when we fit all the data points in our synthesized Type 1 relation that lie in the size range investigated in Larson's 1981 paper, we (somewhat fortuitously) recover Larson's original result of $a_{1}=0.38$. We also plot a density-size relation assuming virial equilibrium and find $n \propto R^{-1.2}$. Furthermore, the dispersion in our simulations is similar to the scatter in Larson's original data set.

\subsection{Speculations on the Cause of Coherence}

There are a surprising number of candidate processes to consider as the cause of coherence in dense cores, many of which can be at work simultaneously. Table 4 summarizes the length scales that are physically meaningful to compare with $R_{\text {coh }}$, and its calculations and implications are discussed below.

\subsubsection{Alfvén Wave Cutoff}

The size scale $R_{\text {coh }}$ at which we find transitions to coherence in low-mass dense cores may be very close to the scale below which no Alfvén waves can propagate in molecular clouds. In a low-ionization medium, Alfvén waves cannot propagate when the neutral-ion collision frequency in the neutral medium (i.e., the rate at which a given neutral encounters ions) is comparable to or less than the wave frequency. Thus, one can define a cutoff wavenumber $k_{\text {cut }}$ beyond which wave motion is not possible (see McKee et al. 1993 and references therein; McKee \& Zweibel 1995). If we define a cutoff size scale, $R_{\text {cut }}$, as the radius of a volume that would accommodate one wave of wavelength $\lambda_{\text {cut }}=2 \pi / k_{\text {cut }}$, then

$$
R_{\text {cut }}=\frac{\lambda_{\text {cut }}}{2}=\frac{\pi v_{\mathrm{A}}}{2 f_{\mathrm{ni}}}
$$

where $v_{\mathrm{A}}$ is the Alfvén speed in gas of density $\rho$,

$$
v_{\mathrm{A}}=\frac{B}{\sqrt{4 \pi \rho}},
$$

and $f_{\mathrm{ni}}$ is the neutral-ion collision rate given by

$$
f_{\mathrm{ni}}=x_{i} \rho \gamma
$$

where $x_{i}$ is the ionization fraction $\left(\rho_{i} / \rho\right)$ and $\gamma$ is the drag coefficient corresponding to neutral-ion collisions. Draine, Roberge, \& Dalgarno (1983) have estimated $\gamma=3.5 \times 10^{13}$ $\mathrm{cm}^{3} \mathrm{~g}^{-1} \mathrm{~s}^{-1}$ for molecular neutral-ion collisions dominated by the dipole moment induced in the neutral (typically $\mathrm{H}_{2}$ ) by the passing ion (typically $\mathrm{HCO}^{+}$).

In estimating the numerical value of $R_{\text {cut }}$ in star-forming regions, the two largest uncertainties are (1) the ionization fraction and (2) the applicability of the "smooth medium" calculations (which give eq. [11]) to the presumed wispy filamentary chaff that surrounds dense cores. If we assume that the ionization in the gas in and around dense cores is produced primarily by cosmic rays, then $x_{i} \approx 10^{-7}$ (Shu,

\begin{tabular}{|c|c|c|c|}
\hline Length Scale & Expression & $\begin{array}{l}\text { Estimate } \\
\quad(\mathrm{pc})\end{array}$ & Comments \\
\hline Alfvén wave cutoff............ & $R_{\mathrm{cut}}=\frac{\pi^{1 / 2}}{4} \frac{B}{\rho^{3 / 2} x_{i} \gamma}$ & 0.3 & $\sim R_{\mathrm{coh}}$ \\
\hline $\begin{array}{c}\text { Inner scale of incompressible } \\
\text { turbulent flow ............... }\end{array}$ & $\lambda_{0} \approx\left[\frac{n_{L} \Delta v_{L} \sigma_{n n}}{(k T / \mu)^{1 / 2} \mathscr{R}_{\mathrm{cr}}}\right]^{-3 / 4} L^{1 / 4}$ & $8 \times 10^{-3}$ & $\begin{array}{l}L=100 \mathrm{pc} ; n_{L}=50 \mathrm{~cm}^{-3} ; \\
\Delta v_{L}=8 \mathrm{~km} \mathrm{~s}{ }^{-1} ; T=15 \mathrm{~K} \\
\mathscr{R}_{\mathrm{cr}}=1000 ; \\
\sigma_{n n}=6 \times 10^{-16} \mathrm{~cm}^{-2}\end{array}$ \\
\hline $\begin{array}{c}\text { Thermal and nonthermal } \\
\text { motions equal .......... }\end{array}$ & $R_{\mathrm{TNT}}=\sqrt{\frac{90}{\mathrm{G}} \frac{k T}{\mu B}}$ & 0.2 & $\begin{array}{l}\sim R_{\text {con }} ; \text { assuming magnetic, } \\
\quad \text { kinetic and gravitational } \\
\text { equipartition }\end{array}$ \\
\hline $\begin{array}{l}\text { Ambipolar diffusion in a } \\
\text { smooth medium ......... }\end{array}$ & $R_{\mathrm{AD}} \approx R_{\mathrm{TNT}}$ & 0.2 & $\begin{array}{l}\sim R_{\text {coh }} ; \text { assuming } \\
\quad \text { predominantly cosmic ray } \\
\quad \text { ionization }\end{array}$ \\
\hline Jeans instability .............. & $R_{\mathrm{J}}=\left(\frac{\pi k T}{4 \mathrm{G} \mu^{2} n}\right)^{1 / 2}$ & 0.2 & $\sim R_{\mathrm{coh}}$ \\
\hline
\end{tabular}

TABLE $4^{\mathrm{a}}$

LENGTH SCALES

${ }^{\mathrm{a}}$ For the conditions: $n=5 \times 10^{3} \mathrm{~cm}^{-3} ; B=20 \mu \mathrm{G} ; T=10 \mathrm{~K} ; \mu=2.33 \mathrm{amu} ; \Delta v=1 \mathrm{~km} \mathrm{~s}^{-1} ; x_{i}=10^{-7}$, unless otherwise stated. 
Adams, \& Lizano 1987) for gas with $n \sim 10^{4}$. Measurements of the Zeeman effect in the $\mathrm{OH}$-emitting gas in and/or around dense cores give magnetic field strengths $B \sim 20-30 \mu \mathrm{G}$ (e.g., $27 \mu \mathrm{G}$ has been measured as the line-ofsight field at the peak of B1; Goodman et al. 1989). Thus, if $n=5 \times 10^{3} \mathrm{~cm}^{-3}, B=20 \mu \mathrm{G}, x_{i}=10^{-7}$, and $\gamma=3.5 \times 10^{13} \mathrm{~cm}^{3} \mathrm{~g}^{-1} \mathrm{~s}^{-1}$, then $R_{\text {cut }}=0.3 \mathrm{pc}$. Notice that this estimate ultimately depends on density, field strength, and the ionization fraction (see Table 4). The ionization fraction in turn depends on the neutral density, the filling factor of the gas, the cosmic-ray flux, and the local flux of ultraviolet photons (see Myers \& Khersonsky 1995), all of which are poorly known. Note that if the relevant density for the conditions quoted above is actually $10^{4}$, then $R_{\text {cut }}$ only goes down to $0.1 \mathrm{pc}$, but if the ionization fraction is as high as $10^{-6}$, then $R_{\text {cut }}$ goes down to 0.03 pc. Taking "reasonable" parameters, though, it is certainly plausible that $R_{\mathrm{coh}} \approx R_{\mathrm{cut}}$.

Before we conclude that inefficient wave coupling offers a great explanation for the transition to coherence, we must point out that the formulation of the cutoff wavelength in equation (11) only considers neutral-ion coupling in the gas. When very small dust grains are charged, they can transmit the effects of the magnetic field to the gas, even in very high gas density regions. In cases where the power-law spectrum of dust grain sizes, $N(a) \propto a^{-x}$, has a large exponent (e.g., $x \approx 3.5$; Mathis, Rumpl, \& Nordsieck 1977), the number of small grains is very large, and the coupling of the gas to charged dust particles can shift $R_{\text {cut }}$ to scales many orders of magnitude smaller than a dense core (Elmegreen \& Fiebig 1993). In the interiors of dense cores, however, there are many reasons to expect that small dust grains are systematically underabundant (e.g., $x \approx 2.8$; Mathis \& Whiffen 1989; Goodman et al. 1995 and references therein), and thus that neutral-ion collisions in the gas phase are what couples the field to the gas and in turn that the calculation in equations (11)-(13) is appropriate.

\subsubsection{Eddies in a Turbulent Flow}

Even without magnetic fields, a natural inner scale $\lambda_{0}$ will arise in a fully developed turbulent flow (see, for example, Landau \& Lifshitz 1959). The inner scale corresponds to the smallest eddy that persists in the case in which energy is injected on the largest scale $L$. (The "inertial subrange" is defined as the range of sizes smaller than $L$ but larger than $\lambda_{0}$.) The "inner scale" for an incompressible flow is given roughly by (see Shu 1992)

$$
\lambda_{0} \sim\left(\frac{\mathfrak{R}}{\mathfrak{R}_{\mathrm{cr}}}\right)^{-3 / 4} L,
$$

where $\mathfrak{R}$ is the Reynolds number of the gas, given by

$$
\mathfrak{R}=\frac{v_{\text {flow }} L}{v},
$$

with $v$ representing the viscosity

$$
v \sim \sigma_{T} l
$$

where $l$ is the mean free path,

$$
l=\frac{\sigma_{T}}{f_{\mathrm{nn}}},
$$

and $f_{\mathrm{nn}}$ is the neutral-neutral collision rate. The "critical
Reynolds number" $\mathfrak{R}_{\mathrm{cr}}$ used in equation (14) is of order $10^{2}$ or $10^{3}$ for viscous shear flows (Shu 1992).

Given all of the approximations in equations (14)-(17) and the fact that the ISM is compressible, it is very difficult to offer a meaningful value of $\lambda_{0}$. But, to make a ballpark estimate of $\lambda_{0}$, we can assume that $L=100$ pc (representative size of a cloud complex or "giant molecular cloud") and that $v_{\text {flow }}=\Delta v_{L} \approx 8 \mathrm{~km} \mathrm{~s}^{-1}$ (a line width representative of the magnitude of the macroscopic motions on the $100 \mathrm{pc}$ scale). The latter assumption causes equation (15) to reduce to

$$
\mathfrak{R}=\frac{L f_{\mathrm{nn}} \Delta v}{\sigma_{T}^{2}},
$$

after use of equations (16) and (17). The neutral-neutral collision rate is given by $f_{\mathrm{nn}}=n \sigma_{T} \sigma_{\mathrm{nn}}$ and can be estimated by assuming a geometric cross section, $\sigma_{\mathrm{nn}}=\left(2 \pi r_{\mathbf{H}_{2}}^{2}\right) \approx 6$ $\times 10^{-16} \mathrm{~cm}^{2}$. For the density on the $100 \mathrm{pc}$ scale, we can assume $n_{L}=50 \mathrm{~cm}^{-3}$ so that using $\Delta v_{L}=8 \mathrm{~km} \mathrm{~s}^{-1}, f_{\mathrm{nn}}=7$ $\times 10^{-10} \mathrm{~s}^{-1}$, and $T=15 \mathrm{~K}$ in equation (18) gives $\mathfrak{R} \approx 3 \times 10^{8}$. Thus, equation (14) gives $\lambda_{0}=8 \times 10^{-3} \mathrm{pc}$ for $\mathfrak{R}_{\text {cr }}=1000$ (see also Table 4). This "cutoff" is of at least an order of magnitude smaller than $R_{\mathrm{coh}}$, but it is only a gross estimate for nonmagnetic turbulence in an incompressible gas.

Simulations of incompressible turbulence in non-selfgravitating unmagnetized fluids (Frisch \& Orszag 1990) show filamentary structures that appear similar to dark clouds. In addition, those structures can and often do contain regions of reduced vorticity which can be associated with coherent structures for scales less than $\lambda_{0}$. Morphologically, Figure 10 looks very similar to the turbulence simulations that give rise to these structures. In turbulence simulations that include the effects of self-gravity, VázquezSemadeni, Passot, \& Pouquet (1996) also find evidence for a reduction in vorticity in high-density clumps formed when colliding regions produce self-gravitating pieces of gas.

In reality, the ISM is significantly magnetized and compressible. In such a medium, the concept of an "eddy" is somewhat inappropriate, so it is hard to calculate a direct "magnetic" analog of $\lambda_{0}$. Nonetheless, the work of Myers \& Khersonsky (1995) shows that the magnetic Reynolds number $\mathfrak{R}_{M}$ is of order 1500 times smaller than $\mathfrak{R}$ in a dense core environment. In general, given the form of equation (14), we can see that this reduction in Reynolds number would tend to give a larger inner scale in a magnetized turbulent flow, as compared with a nonmagnetized flow, by a factor of very roughly $1500^{3 / 4} \sim 200$. This quick estimate and the cutoff wavelength discussion of $\S 5.3 .1$ suggest that the analog of $\lambda_{0}$ in a magnetized, compressible, medium should be significantly larger than $\lambda_{0}$ and thus perhaps more commensurate with $R_{\text {coh}}$.

\subsubsection{The Most Likely Scenario: A Dissipation Threshold}

Given that the turbulence in the ISM is magnetized, the eddy picture of $\S 5.3 .2$ and the Alfvén wave picture of $\S 5.3 .1$ can be unified into a single "dissipation threshold" scenario. In this view, at scales larger than $R_{\text {coh }}$, some source of nonthermal (i.e., Alfvén wave or turbulent) pressure is available. The reduction in available pressure on the coherence scale is caused by dissipation. For sub-Alfvénic MHD disturbances, the dissipation is caused by reduced neutral-ion coupling and by some shocks caused by supersonic motions along field lines. For super-Alfvénic motions, radiative 
shocks will also cause significant dissipation. We expect that when the dissipation reaches the threshold at which the wavelike and turbulent motions in the magnetized gas are substantially quenched, the observed value of $R_{\text {coh }}$ is reached. From that point on, other physical processes dominate the formation of structures on scales smaller than $R_{\text {coh }}$ (e.g., "static" processes like those discussed in $\S 5.3 .4$ below).

At present, it is just becoming possible to make numerical models that might bolster or discredit this scenario. Gammie, Ostriker, and Stone are modeling regions like the ones discussed here, and their early results (C. Gammie, E. Ostriker, \& J. Stone 1997, private communication) predict that Alfvén wave-like disturbances will indeed pervade the ISM and can support self-gravitating molecular clouds, when adequately driven (Gammie \& Ostriker 1996). In the simulations carried out thus far, ideal MHD, or $x_{i}=1$, has been assumed, so information about a cutoff scale cannot be extracted. The next generation of simulations will allow for neutral-ion slippage and should be able to find any dissipation thresholds and/or coherence scales that may be present.

\subsubsection{Explanations More Applicable to a Static Medium}

Two static approximations of $R_{\text {coh }}$ can also be made. If we treat the gas under study as smoothly varying in density, then we can apply both ambipolar diffusion calculations (MG88b; Lizano \& Shu 1989; Mouschovias 1991) and the Jeans analysis. Both these analyses give "transitions" close to the observed values of $R_{\text {coh }}(\sim 0.1 \mathrm{pc})$ when initial conditions typical of the outer parts of a dense core are used (Table 4). But, because there is no natural reason to select these particular initial conditions a priori, these analyses may be more applicable to a region in which a predecessor to a coherent core has already formed, perhaps as a result of the dissipation threshold process described in $\S$ 5.3.3.

Ambipolar Diffusion.-The estimate for $R_{\text {cut }}$ in $\S 5.3 .1$ depends sensitively on the ionization fraction in the gas and describes the ability of the neutrals to couple to the field in wavelike motions. There is another size scale estimate that involves neutral-ion slippage but in a core without waves. In such a case, the " ambipolar diffusion length scale" $R_{\mathrm{AD}}$ is used to describe the point at which magnetic forces are insufficient to counter a core's tendency toward gravitational collapse. It is readily apparent that ambipolar diffusion - which allows neutrals to slide past ions - causes the mass-to-flux ratio to increase toward the center of a core, but the degree to which it does so depends on the exact relation between the ionization fraction and the neutral density. This ionization-density dependence and the role of charged grains (see $\S$ 5.3.1) are still the subjects of great debate (e.g., see Lizano \& Shu 1989; Mouschovias \& Morton 1991; Nishi, Nakano, \& Umebayashi 1991; Ciolek \& Mouschovias 1993; Basu \& Mouschovias 1994; Ciolek \& Mouschovias 1994; Morton, Mouschovias, \& Ciolek 1994; Basu \& Mouschovias 1995b, 1995a; Ciolek \& Mouschovias 1995; Myers \& Khersonsky 1995). In general, studies that rely on gas particles as the only relevant charge-carriers in dense cores and cosmic rays as the only source of ionization estimate $R_{\mathrm{AD}}$ to be of order $0.1 \mathrm{pc}$. Studies that allow for significant numbers of very small charged grains (Ciolek \& Mouschovias 1993, 1994) or some UV ionization (Myers \& Khersonsky 1995) inside dense cores find $R_{\mathrm{AD}}$ to be as much as 10 times smaller.
In Table 4, we give a very simple estimate of $R_{\mathrm{AD}}$, which is calculated considering (1) only gaseous neutral-ion interactions and (2) cosmic rays as the only source of ionization. In that case, the ion fraction is simply proportional to the inverse of the square root of the neutral density. As a result, the length scale for efficient ambipolar diffusion is ultimately set by the density-size relation (see MG88b, eqs. [16][18]). With $R_{\mathrm{AD}}$ defined as the radius at which the ambipolar diffusion time is at a minimum, the relevant break in slope of the density-size relation occurs at the radius at which thermal and nonthermal motions are equal. This radius, $R_{\mathrm{TNT}}$ (so-named by Myers, Ladd, \& Fuller 1991), is also listed in Table 4 for the case in which the nonthermal motions are magnetic in origin, and magnetic, kinetic, and gravitational forces are in equipartition (MG88b).

For conditions relevant to a dense core environment (Table 4 ), $R_{\mathrm{AD}} \approx R_{\mathrm{TNT}}=0.2$ pc. We must reemphasize, though, that the relevant transition to coherence may be the one that determines how those environmental conditions came to be. The transitions discussed in $\S 5.3 .3$ are likely to be most relevant in producing those conditions.

Jeans Analysis.-If the line width became purely thermal inside of $R_{\text {coh }}$ and the cloud were of a uniform density $\rho_{0}$, then the Jeans length

$$
R_{\mathrm{J}}=\frac{\lambda_{\mathrm{J}}}{2}=\left(\frac{\pi \sigma_{T}^{2}}{4 G \rho_{0}}\right)^{1 / 2}
$$

should be comparable to $R_{\text {coh }}$. As shown in Table 4, using $T=10 \mathrm{~K}$ and $\rho_{0}=5 \times 10^{3} \mathrm{~cm}^{-3}$, equation (19) gives $R_{\mathrm{J}}=$ $0.2 \mathrm{pc}$, which is very close to the values found for $R_{\text {coh }}$. Recent work by Adams, Fatuzzo, \& Watkins (1994) has shown that when a Jeans-type analysis is applied to more realistic initial conditions (with density inhomogeneities and magnetic field pressure), the preferred scale for fragmentation remains within a factor of 2 of the traditional Jeans length. When one allows for the existence of a central condensation in a self-gravitating smooth medium, the isothermal mass on the verge of collapse is given by the radius of a critical Bonner-Ebert sphere, not the Jeans length (McLaughlin \& Pudritz 1996). Mouschovias (1991) points out that the radius of this sphere is $R_{\mathrm{J}} / 1.5$, which is still of $\operatorname{order} R_{\mathrm{J}}$. Thus, it may still be quite relevant that $R_{\mathrm{J}} \approx R_{\text {coh }}$, despite the density structure and (small) residual nonthermal line width present in realistic clouds.

Again, though, we emphasize that it is only when Jeanstype instability analyses are applied to conditions relevant to the environments of dense cores that they give fragment masses comparable to the cores themselves. Much more massive regions would have very supersonic line widths or would be collapsing, either of which makes the traditional Jeans analysis inapplicable. In fact, on scales much larger than $R_{\text {coh }}$, the Jeans analysis predicts fragments that are much larger than cores (see eq. [19]). Thus, we maintain the hypothesis that more dynamic processes set the scale for the coherent regions that can ultimately break into identifiable Jeans-mass size pieces.

\section{FOOD FOR THOUGHT}

\subsection{Unanswered Questions}

6.1.1. How Much Structure Is there inside a Coherent Dense Core?

Scientists are perpetually being fooled into believing that they have found the "fundamental building block" in one 
process or another. Stars clearly represent a fundamental building block in astronomy, but coherent dense cores probably do not. Even though they give rise to stars, the cores do not just each form a single star-some form one, some form a handful, and some may form none. The question to be answered next is, How much substructure (if any) is there inside dense cores that is not associated with warm or dense regions around individual protostars? Early results based on $\mathrm{NH}_{3}$ VLA-mapping of 11 dense cores (Goodman et al. 1998) indicate that the answer to this question is "not much": almost all of the substructure seen inside dense cores is associated with individual protostars or outflows from those stars (e.g., Tafalla \& Bachiller 1995). In other words, most of the flux from cold dense gas tracers like $\mathrm{NH}_{3}$ seems to originate in a relatively smooth distribution extended throughout the high-resolution maps and is thus undetectable by interferometers that are only sensitive to high-spatial-frequency structure.

\subsubsection{Is the Residual Nonthermal Line Width Evident in $\mathrm{NH}_{3}$} Observations "Inside" the Core or in an "Envelope"?

The careful reader will have noticed by now that the ensemble of Type 4 line width-size relations shown in Figure 12 and Figure $9 f$ overlap each other in radius coverage. Figure 14 illustrates why this is so. Low-density tracers (e.g., line of sight A in Fig. 14) can sample many size scales, down to a minimum spatial scale that corresponds to the maximum density they are capable of tracing (see discussion in $\S 5.2$ ). Higher density tracers, such as $\mathbf{N H}_{3}$, which are excited over smaller volumes, also trace a limited range of density, which can severely restrict the range of size scales they can trace if the density is only rarely high enough to excite them (i.e., they are excited only in cores, or in tiny high-density fluctuations in the chaff; see Schneider et al. 1996). In Figure 14, line of sight B passes through the outskirts of a core, where $\mathrm{NH}_{3}$ emission is (hypothetically) produced all the way through the core (as at $r_{3}$ in Fig. 4). However, lines of sight through the densest, coldest portions of the core (e.g., line of sight C) may contain emission from only an "envelope" (defined by $R_{\text {in }}<R<R_{\text {out }}$ ) of $\mathrm{NH}_{3}$ around gas too dense to emit strongly in $\mathrm{NH}_{3}$ (at $R<R_{\text {in }}$ ).

In other words, the use of a given probe introduces a density selection effect into a data set, which restricts the range of size scales to which each density tracer is sensitive. Tracers do not turn on and off abruptly at particular density boundaries, so the range of size scales to which each tracer is sensitive will overlap to some degree. As a result of this overlap, when we convert from radius as projected on the sky to size scale in a line width-size relation, the twodimensional to three-dimensional mapping is imperfect: emission from lower density gas is always superposed on emission from higher density gas (see lines of sight B or C in Fig. 14).

We are thus left with the question, Where does the nonzero nonthermal line width in $\mathrm{NH}_{3}$ cores come from?

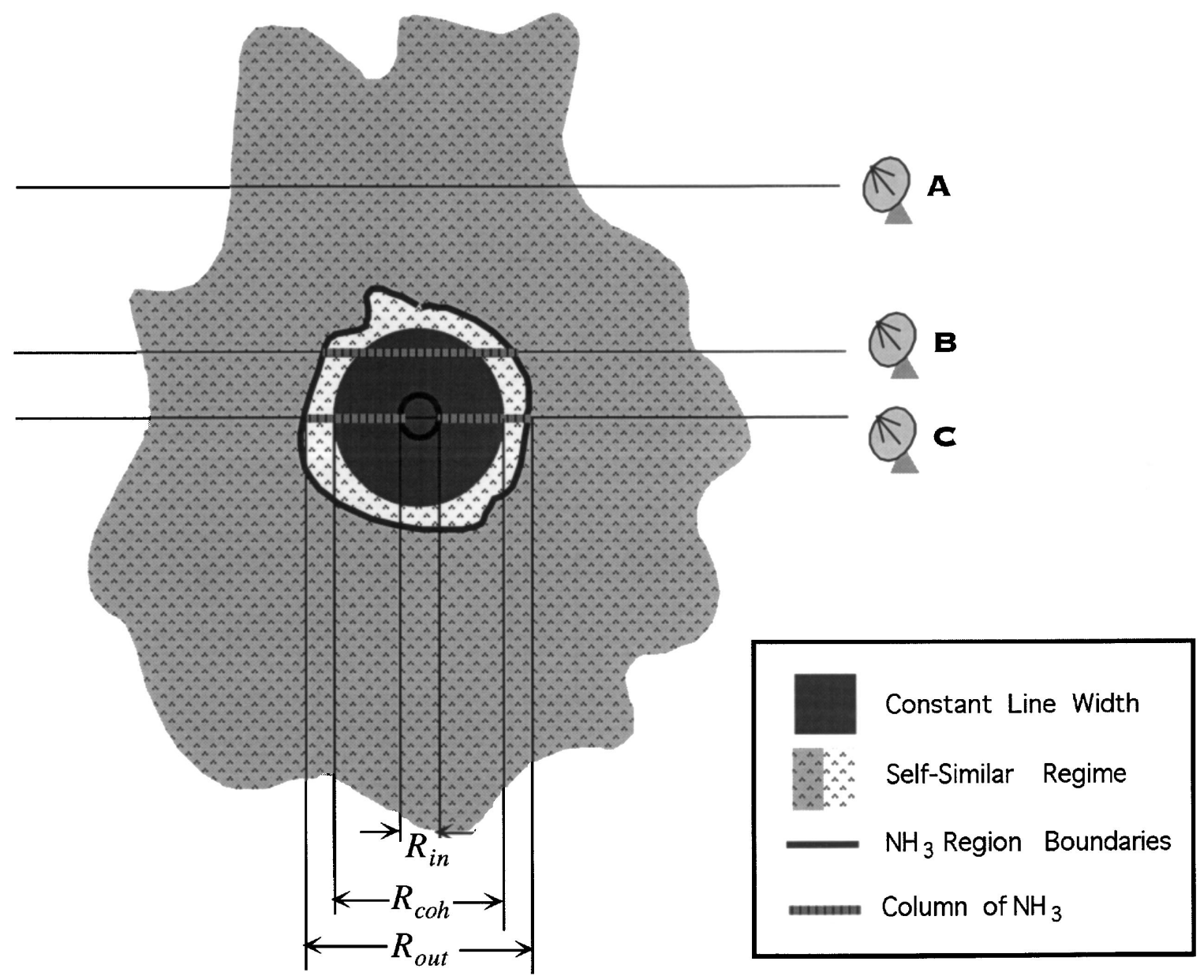

FIG. 14.-A coherent dense core and its environment. Emission from dense gas as traced by $\mathrm{NH}_{3}$ may arise only in a shell where $R_{\text {in }}<R<R_{\text {out }}$. We have shown that $R_{\text {in }}<R_{\text {coh }}<R_{\text {out }}$, but the exact relation between $R_{\text {coh }}, R_{\text {in }}$, and $R_{\text {out }}$ remains to be determined. 
Does it come from the entire core volume or just from a shell-like volume of gas at densities near $10^{4} \mathrm{~cm}^{-3}$ ? Chemical modeling by Kuiper et al. (1996) suggests that $\mathrm{NH}_{3}$ is a "late-time" molecule, in that it exists in the oldest, interior, regions of cores. In contrast, CS and CCS are thought to be "early-time" molecules that only exist for a limited time after being glommed onto the core from the chaff. This modeling suggests that the $\mathrm{NH}_{3}$ emission originates in more of a filled volume than a shell. Our finding in $\S 4.2 .4$ that the column density inside cores rises rapidly with decreasing radius also argues against $\mathrm{NH}_{3}$ being shell-like in three dimensions. From the analysis in this paper, we can say that $R_{\text {out }}>R_{\text {coh }}>R_{\text {in }}$, but we cannot say exactly how much less $R_{\text {in }}$ is than $R_{\text {coh }}$. Higher resolution multitransition $\mathrm{NH}_{3}$ observations, which could reveal very subtle limb-brightening in plots of the two-dimensional projection of the three-dimensional distribution of $\mathrm{NH}_{3}$ molecules, might be able to settle this question in the future.

One other relevant piece of evidence in this matter comes from a recent study of dense cores by Butner, Lada, \& Loren (1995). In that study, the authors found that $\mathrm{DCO}^{+}$ line widths were consistently higher than $\mathrm{NH}_{3}$ widths, even though the extent of the $\mathrm{DCO}^{+}$emission is similar to the $\mathrm{NH}_{3}$. The authors suggest that chemical effects may be playing a role in this difference. We agree and suggest that it is easily possible that the $\mathrm{DCO}^{+}$is excited in more of a shell-like geometry (see Fig. 14) than the $\mathrm{NH}_{3}$ and is thus associated with a larger size scale along the line of sight than its plane-of-the sky extent might otherwise imply (see Fig. 4). Measuring the "size" of $\mathrm{DCO}^{+}$cores directly, on the plane of the sky, would then systematically assign too small a size scale to the (larger than $\mathbf{N H}_{3}$ ) line widths observed.

If we accept that the residual nonthermal line width does persist throughout the volume of a dense core, we are still left with a question about its origin. It seems it must represent some new regime in either the turbulent or wavelike properties of the velocity field in the gas. Some (e.g., F. H. Shu 1997, private communication) have suggested that this kind of residual width might be due to subsonic turbulence within cores, which is driven by processes on larger scales.

\subsubsection{What about High-Mass Star-forming Regions?}

The nearest massive star-forming region (Orion) is 3 times farther away than the nearest low-mass star-forming regions. As a result, it is harder to identify blobs of dense gas similar to the low-mass cores discussed in this paper in massive star-forming regions. The cores that are identified in massive star-forming regions typically have much larger masses, sizes, and line widths than a typical low-mass core (Wouterloot, Walmsley, \& Henkel 1988; Harju, Walmsley, \& Wouterloot 1991, 1993; Lada et al. 1991; Tatematsu et al. 1993; Ladd, Myers, \& Goodman 1994; Cesaroni \& Wilson 1994; Caselli \& Myers 1995). These cores are typically each associated with several forming stars, and it is virtually impossible to pick out individual "blobs" in molecular-line maps that might represent the analog to the coherent dense cores in low-mass star-forming regions. It is, however, relatively easy to identify such blobs in submillimeter continuum maps (e.g., P. André 1996, private communication). Thus, if in the future we can devise a Doppler velocitysensitive technique to pick out only the gas associated with a submillimeter-identified core, we might be able to look a signature of coherence in high-mass star-forming regions.
6.1.4. Is it Possible to Identify Potential Precursors to Dense Cores from the Velocity Field in Lower Density Gas?

It is possible that the locations of today's cores correspond to what once were simply "eddies" or regions of reduced kinetic energy in the larger scale turbulent flow of the ISM. In that case, when the average density of the turbulent gas is close to that needed for gravitational collapse and the downward fluctuation in kinetic energy is sufficiently long-lived, the fluctuation should be able to initiate the slow collapse of an eddy into a coherent dense core. If the low kinetic energy region is formed in a shock, rather than just an ephemeral eddy, it will also have a higher than average density, which will make it even more likely to become coherent. If this scenario is realistic and precursors to coherent regions can be identified in molecular-line databases (e.g., Magnani, LaRosa, \& Shore 1993), we should be able to learn more about the process that creates an eddy, forms a core, and then forms a star. Analyses that decompose spectra into multiple line-of-sight components and analyze the spatial relations among velocity components might be able to identify potential future cores-as regions of spatially correlated, low line width, low velocity gradient gas. There is some hope that such an analysis could produce a "core-forming gas" IMF. Such an IMF might be more relevant for comparison with the stellar IMF than the many of the "clump" IMFs used currently.

\subsection{Synopsis}

We have undertaken a new investigation into the nature of the line width-size relations. We find that the power-law slope of single-tracer line width-size relations gets progressively shallower toward higher densities, and we interpret this result as a transition to coherence. Our specific findings are as follows:

1. Maps of the $\mathrm{OH}$ and $\mathrm{C}^{18} \mathrm{O}$ emission from the $n \sim 10^{3}$ $\mathrm{cm}^{-3}$ gas around dense cores show FWHM line width decreasing toward antenna temperature peaks. By fitting antenna temperature as a function of size and line width as a function of antenna temperature, we deduce line widthsize relations for $\mathrm{OH}$ maps of $\mathrm{B} 1$ and $\mathrm{TMC}-1 \mathrm{C}$ and $\mathrm{C}^{18} \mathrm{O}$ map of L1251A. The power-law slopes in these single-tracer single-cloud line width size relations are typically $a_{4} \sim 0.2$.

2. $\mathrm{NH}_{3}$ maps of dense cores within the regions mapped in $\mathrm{OH}$ and $\mathrm{C}^{18} \mathrm{O}$ show an even shallower dependence of line width on size, with power-law slope $a_{4} \sim 0.1$ in fits to the full core and slope $a_{4} \sim 0$ within the half-power contour of the core (see Paper I). We interpret the decrease in slope between the lower density regions $\left(\mathrm{OH}\right.$ and $\mathrm{C}^{18} \mathrm{O}$ maps) and the higher density regions $\left(\mathrm{NH}_{3}\right.$ maps) as a transition to coherence.

3. The value of the nonthermal line width at which coherence is established is always less than but still of the order of the thermal line width of $\mathrm{H}_{2}$. In other words, the "turbulent" component of the line width in these cores is always observed to be subsonic. This small residual width means coherent cores are similar to, but not exactly the same as, isothermal balls of gas.

4. The $\mathrm{NH}_{3}$ data indicate a much steeper dependence of column density on size $\left(N \propto R^{-0.9}\right)$ than do the $\mathrm{OH}$ or the $\mathrm{C}^{18} \mathrm{O}$ data $\left(N \propto R^{-0.2}\right)$. This may imply that the filling factor within the $\mathrm{NH}_{3}$ cores is larger than in their surroundings.

5. Given (2) and (4), we put forth the hypothesis (see Fig. 
10) that the molecular clouds which contain low-mass dense cores are self-similar in nature on size scales larger than $R_{\text {coh }}$, and that within $R_{\text {coh }}$, cores are coherent, in that their filling factor is large and they are characterized by a very small, roughly constant, velocity dispersion.

6. We place our study and previous studies of line widthsize relations into context by defining four Types of line width-size relations and discussing the operational and physical differences among them. The Types and their most important features are as follows:

Type 1: Multitracer, multicloud intercomparison.Shows the (cosmic) scatter about an overall line width-size relation.

Type 2: Single-tracer, multicloud intercomparison.Heavily influenced by the column density-tracing properties of probe used.

Type 3: Multitracer study of a single cloud.-Good for studying density profile of individual cores and their environments.

Type 4: Single-tracer study of a single cloud.-Gives best idea of dynamics within a particular density regime.

Through Monte Carlo simulations, we find that a realistic mixture of Type 3 relations can reproduce the original Type 1 relation uncovered by Larson (1981). Figure 12 demonstrates how the data presented in this paper can be used to create each of the four distinct Types of line widthsize relation. The figure illustrates that the slope derived for each Type need not be equal.
7. We point out that the transition to coherence takes place at $R_{\text {coh }} \sim 0.1 \mathrm{pc}$, which is just a bit larger than the size scale $(0.04 \mathrm{pc})$ at which Larson (1995) has found a transition in the power-law slope of the stellar clustering function in Taurus. These results are consistent with a scenario in which the distribution of gas and stars on scales $>R_{\text {coh }}$ is determined by a self-similar process (such as MHD turbulence), and fragmentation dominates on smaller scales.

8. The transition to coherence is likely produced when the conditions in the gas cross a threshold at which the forces responsible for maintaining the turbulence and/or waves on larger scales can no longer operate. The forces responsible are likely to rely on the coupling of magnetic fields to the neutral gas, and the critical conditions producing the transition consist of a critical combination of low temperature, high density, and low ionization fraction.

We would like to thank Fred Adams, Peter Barnes, Paola Caselli, Bruce Elmegreen, Gary Fuller, Charles Gammie, Ned Ladd, Bill Langer, Richard Larson, Steve Lubow, Chris McKee, Phil Myers, Steve Stahler, Jim Stone, Enrique Vázquez-Semadeni, and Taoling Xie for their insightful comments on this work as it was evolving. We especially thank Eve Ostriker, the referee, for many helpful comments and suggestions. A. G. is grateful to the Osservatorio Astrofisico di Arcetri for providing the hospitable atmosphere where the final revisions to this paper were made.

\section{REFERENCES}

Adams, F. C., Fatuzzo, M., \& Watkins, R. 1994, ApJ, 426, 629

Arons, J., \& Max, C. E. 1975, ApJ, 196, L77

Barranco, J. A., \& Goodman, A. A. 1998, ApJ, 504, 207 (Paper I)

Basu, S., \& Mouschovias, T. Ch. 1994, ApJ, 432, 720 1995a, ApJ, 452, 386

. 1995b, ApJ, 453, 271

Benson, P. J., \& Myers, P. C. 1989, ApJS, 71, 89

Bergin, E. A., Snell, R. L., \& Goldsmith, P. F. 1996, 460, 343

Butner, H. M., Lada, E. A., \& Loren, R. B. 1995, ApJ, 448, 207

Carlberg, R. G., \& Pudritz, R. E. 1991, MNRAS, 247, 353

Caselli, P., \& Myers, P. C. 1995, ApJ, 446, 665

Cesaroni, R., \& Wilson, T. L. 1994, A\&A, 281, 209

Ciolek, G. E., \& Mouschovias, T. Ch. 1993, ApJ, 418, 774 1994, ApJ, 425, 142

.1995, ApJ, 454, 194

Crutcher, R. M., Mouschovias, T. Ch., Troland, T. H., \& Ciolek, G. E. 1994, ApJ, 427, 839

Crutcher, R. M., Troland, T. H., Goodman, A. A., Kazès, I., Heiles, C., \& Myers, P. C. 1993, ApJ, 407, 175

Dame, T. M., Elmegreen, B. G., Cohen, R. S., \& Thaddeus, P. 1986, ApJ, 305,892

Draine, B. T., Roberge, W. G., \& Dalgarno, A. 1983, ApJ, 264, 485

Dubinski, J., Narayan, R., \& Phillips, T. G. 1995, ApJ, 448, 226

Elmegreen, B. G., \& Falgarone, E. 1996, ApJ, 471, 816

Elmegreen, B. G., \& Fiebig, D. 1993, A\&A, 270, 397

Falgarone, E., Lis, D. C., Phillips, T. G., Pouquet, A., Porter, D. H., \& Woodward, P. R. 1994, ApJ, 436, 728

Falgarone, E., \& Phillips, T. G. 1990, ApJ, 359, 344

Falgarone, E., Puget, J.-L., \& Pérault, M. 1992, A\&A, 257, 715

Fleck, R. C. J. 1988, ApJ, 328, 299

Frisch, U., \& Orszag, S. A. 1990, Phys. Today, January 1990, 24

Fuller, G. A., \& Myers, P. C. 1992, ApJ, 384, 523

Gammie, C., \& Ostriker, E. 1996, ApJ, 466, 814

Gomez, M., Hartmann, L., Kenyon, S. J., \& Hewett, R. 1993, AJ, 105, 1927

Goodman, A. A., Benson, P. J., Fuller, G. A., \& Myers, P. C. 1993, ApJ, 406, 528

Goodman, A. A., Benson, P. J., Fuller, G. A., Wilner, D. J., \& Zhang, Q. 1998 , in preparation

Goodman, A. A., Crutcher, R. M., Heiles, C., Myers, P. C., \& Troland, T. H. 1989, ApJ, 338, L61

Goodman, A. A., \& Heiles, C. 1994, ApJ, 424, 208
Goodman, A. A., Jones, T. J., Lada, E. A., \& Myers, P. C. 1995, ApJ, 448, 748

Harju, J., Walmsley, C. M., \& Wouterloot, J. G. A. 1991, A\&A, 245, 643 . 1993, A\&ÁS, 98, 51

Heiles, C., Goodman, A. A., McKee, C. F., \& Zweibel, E. G. 1993, in Protostars and Planets III, ed. E. H. Levy \& J. I. Lunine (Tucson: Univ. of Arizona Press), 279

Henriksen, R. N. 1991, ApJ, 377, 500

Heyer, M. H., Carpenter, J. M., \& Ladd, E. F. 1996, ApJ, 463, 630

Heyer, M. H., \& Schloerb, F. P. 1997, ApJ, 475, 173

Kleiner, S. C., \& Dickman, R. L. 1987, ApJ, 312, 837

Kolmogorov, A. N. 1941, Comptes Rendus de l'Academie des Sciences de l'URSS, 30, 301; reprinted in Friedlander, S. K. and Topper, L., eds. 1961. Turbulence: Classic Papers on Statistical Theory (New York: Interscience)

Kuiper, T. B. H., Langer, W. D., \& Velusamy, T. 1996, ApJ, 468, 761

Lada, E. A. 1992, ApJ, 393, L25

Lada, E. A., Bally, J., \& Stark, A. A. 1991, ApJ, 368, 432

Ladd, E. F., Myers, P. C., \& Goodman, A. A. 1994, ApJ, 433, 117

Landau, L. D., \& Lifshitz, E. M. 1959, Fluid Mechanics (Elmsford, NY: Permagon)

Larson, R. B. 1981, MNRAS, 194, 809

1995, MNRAS, 272, 213

Lis, D. C., Pety, J., Phillips, T. G., \& Falgarone, E. 1996, 463, 623

Lizano, S., \& Shu, F. H. 1989, ApJ, 342, 834

Magnani, L., LaRosa, T. N., \& Shore, S. N. 1993, ApJ, 402, 226

Mathis, J. S., Rumpl, W., \& Nordsieck, K. H. 1977, ApJ, 217, 425

Mathis, J. S., \& Whiffen, G. 1989, ApJ, 341, 808

McKee, C. F., \& Zweibel, E. G. 1995, ApJ, 440, 686

McKee, C. F., Zweibel, E. G., Goodman, A. A., \& Heiles, C. 1993, in Protostars and Planets III, ed. E. H. Levy \& J. I. Lunine (Tucson: Univ. of Arizona Press), 327

McLaughlin, D. E., \& Pudritz, R. E. 1996, ApJ, 476, 750

Miesch, M. S., \& Bally, J. 1994, ApJ, 429, 645

Morton, S. A., Mouschovias, T. Ch., \& Ciolek, G. E. 1994, ApJ, 421, 561

Mouschovias, T. Ch. 1991, ApJ, 373, 169

Mouschovias, T. Ch., \& Morton, S. A. 1991, ApJ, 371, 296

Myers, P. C. 1983, ApJ, 270, 105

Myers, P. C., \& Benson, P. J. 1983, ApJ, 266, 309

Myers, P. C., \& Fuller, G. A. 1992, ApJ, 396, 631

Myers, P. C., \& Goodman, A. A. 1988a, ApJ, 326, L27 
Myers, P. C., \& Goodman, A. A. 1988b, ApJ, 329, 392 (MG88b)

Myers, P. C., Goodman, A. A., Güsten, R., \& Heiles, C. 1995, ApJ, 442, 177

Myers, P. C., \& Khersonsky, V. K. 1995, ApJ, 442, 186

Myers, P. C., Ladd, E. F., \& Fuller, G. A. 1991, ApJ, 372, L95

Nishi, R., Nakano, T., \& Umebayashi, T. 1991, ApJ, 368, 181

Passot, T., Vázquez-Semadeni, E., \& Pouquet, A. 1996, ApJ, 455, 536

Pérault, M., Falgarone, E., \& Puget, J. L. 1986, A\&A, 157, 139

Scalo, J. M. 1984, ApJ, 277, 556

1987, in Interstellar Processes, ed. H. Thronson \& D. Hollenbach (Dordrecht: Reidel), 349

Schneider, N., Stutzki, J., Winnewisser, G., \& Blitz, L. 1996, ApJ, 468, L119

Shu, F. H. 1992, The Physics of Astrophysics: Gas Dynamics (Mill Valley, CA: University Science Books)
Shu, F. H., Adams, F. C., \& Lizano, S. 1987, ARA\&A, 25, 23

Solomon, P. M., Rivolo, A. R., Barret, J., \& Yahil, A. 1987, ApJ, 319, 730

Stutzki, J., \& Güsten, R. 1990, ApJ, 356, 513

Tafalla, M., \& Bachiller, R. 1995, ApJ, 443, L37

Tatematsu, K., et al. 1993, ApJ, 404, 643

Vázquez-Semadeni, E., Ballesteros-Paredes, J., \& Rodriguez, L. F. 1997, ApJ, 474, 292

Vázquez-Semadeni, E., Passot, T., \& Pouquet, A. 1996, ApJ, 473, 881

Williams, J., de Geus, E., \& Blitz, L. 1994, ApJ, 428, 693

Wouterloot, J. G. A., Walmsley, C. M., \& Henkel, C. 1988, A\&A, 203, 367

Zhou, S., Wu, Y., Evans, N. J., Fuller, G. A., \& Myers, P. C. 1989, ApJ, 364 168

Zweibel, E. G., \& Josafatsson, K. A. 1983, ApJ, 270, 511 